\title{
A systematic review of structural and functional MRI studies on pain catastrophizing
}

This article was published in the following Dove Press journal:

Journal of Pain Research

\author{
Attila Galambos $\mathbb{D}^{1-3}$ \\ Edina Szabó $\mathbb{D}^{1-3}$ \\ Zita Nagy ${ }^{2}$ \\ Andrea Edit Édes $\mathbb{D}^{4,5}$ \\ Natália Kocsel $\mathbb{D}^{1,2,4}$ \\ Gabriella Juhász (D) ${ }^{4-6}$ \\ Gyöngyi Kökönyei $\mathbb{D}^{2,4,5}$
}

'Doctoral School of Psychology, ELTE Eötvös Loránd University, Budapest, Hungary; ${ }^{2}$ Institute of Psychology, ELTE Eötvös Loránd University, Budapest, Hungary; ${ }^{3}$ MTA-SE

Neuropsychopharmacology and Neurochemistry Research Group, Hungarian Academy of Sciences, Semmelweis University, Budapest, Hungary; ${ }^{4}$ SE-NAP2 Genetic Brain Imaging Migraine Research Group, Hungarian Academy of Sciences, Semmelweis University, Budapest, Hungary; ${ }^{5}$ Department of

Pharmacodynamics, Faculty of Pharmacy, Semmelweis University, Budapest, Hungary; ${ }^{6}$ Neuroscience and Psychiatry Unit, The University of Manchester, Manchester, United Kingdom and Manchester Academic Health Sciences Centre, Manchester, United Kingdom
Correspondence: Gyöngyi Kökönyei Institute of Psychology, ELTE Eötvös Loránd University, Izabella Street 46., Budapest $\mathrm{H}-1064$, Hungary

Tel +36I 46I 2600

Fax +36I 46I 2695

Email kokonyei.gyongyi@ppk.elte.hu
Objectives: Pain catastrophizing is reliably associated with pain reports during experimental pain in healthy, pain-free subjects and in people with chronic pain. It also correlates with self-reports of clinical pain intensity/severity in a variety of disorders characterized by chronic pain in adults, adolescents and children. However, processes, through which it exerts its effects are yet unclear. In this paper, our primary aim was to synthesize neuroimaging research to open a window to possible mechanisms underlying pain catastrophizing in both chronic pain patients and healthy controls. We also aimed to compare whether the neural correlates of pain catastrophizing are similar in these two groups.

Methods: PubMed and the Web of Science were searched for magnetic resonance imaging (MRI) studies that explored neural correlates of pain catastrophizing.

Results: Twenty articles met the inclusion criteria. The results of our review show a connection between pain catastrophizing and brain areas tightly connected to pain perception (including the somatosensory cortices, anterior insula, anterior cingulate cortex and thalamus) and/or modulation (eg, the dorsolateral prefrontal cortex). Our results also highlight that these processes - in relation to pain catastrophizing - are more pronounced in chronic pain patients, suggesting that structural and functional brain alterations (and perhaps mechanisms) related to pain catastrophizing may depend on prior and/or relatively stable/ constant pain experience. However, we also found methodological issues and differences that could lead to divergent results.

Discussion: Based on our results, pain catastrophizing might be related to salience detection, pain processing, and top-down attentional processes. More research is recommended to explore neural changes to specific types of catastrophizing thoughts (eg, experimentally induced and/or state). Furthermore, we provide ideas regarding pain catastrophizing studies in the future for a more standardized approach.

Keywords: pain catastrophizing, DLPFC, anterior insula, chronic pain, neuroimaging

\section{Introduction}

Among cognitive factors, pain catastrophizing, defined as a tendency to magnify and ruminate about pain and having a helpless attitude toward actual or anticipated pain, ${ }^{1}$ is reliably associated with pain reports during experimental pain in healthy pain-free subjects and in people with chronic pain. ${ }^{2}$ Pain catastrophizing affects not just the actual experience of painful stimuli, but it can bias pain recall ${ }^{3}$ even several months after surgery. 4

Pain catastrophizing has been demonstrated to be associated with self-reports of clinical pain intensity/severity in a variety of disorders characterized by chronic pain in adults, for instance in rheumatic diseases, ${ }^{5}$ in low back pain, ${ }^{6}$ in headache ${ }^{7}$ 
and in children and adolescents. ${ }^{89}$ Pain catastrophizing is also associated concurrently and prospectively with pain reports or self-reports of consequences of chronic pain eg, with disability in migraine $e^{10,11}$ and in rheumatic diseases, ${ }^{5}$ loss of work in low back pain ${ }^{12}$ and medication consumption in chronic musculoskeletal pain. ${ }^{13}$ In addition, pain catastrophizing is suggested to have a prognostic value in the maintenance of pain and in the development of chronic pain ${ }^{14,15}$ and has also been demonstrated to influence the success of pharmacological and psychosocial treatments of chronic pain. ${ }^{16-18}$

While pain catastrophizing is associated concurrently and prospectively with pain reports or self-reports of consequences of chronic pain (see above), its relationship with pain threshold is contradictory. ${ }^{19-22}$ Studies on nociceptive flexion reflex threshold (NFR), as an indirect index for spinal nociceptive processing, ${ }^{23}$ consistently reported no relationship between NFR and pain catastrophizing either in healthy subjects ${ }^{19,24,25}$ or in chronic pain patients. ${ }^{21,26}$ Based on these results the question is whether pain catastrophizing simply affects verbal reports of pain, or exerts an influence also on neurobiological (supraspinal) processes to noxious stimuli. Therefore, it is important to identify the mechanisms by which catastrophizing may influence pain perception. Convergent evidence from experimental studies targeting attentional processes suggest that pain catastrophizing may modulate top-down attentional processes, which results in a deficit in attentional disengagement from pain-related information. ${ }^{27-30}$

Neuroimaging has begun to provide evidence that pain-related brain activity is related to pain catastrophizing. The first study that demonstrated that pain catastrophizing relates to pain processing was done in fibromyalgia $(\mathrm{FM}),{ }^{31}$ and then this question was tested in other chronic pain samples and in healthy samples as well. In this paper, our primary aim was to synthesize neuroimaging research to open a window to possible mechanisms underlying pain catastrophizing.

Since pain catastrophizing shows a robust association with perceived/self-reported pain (see above), brain areas implicated in pain perception - especially in the sensory aspect (such as somatosensory areas (both primary, S1 and secondary, S2), posterior insula (pINS), thalamus) and affective/emotional aspect (anterior cingulate cortex (ACC) and anterior insula (aINS)) of pain perception ${ }^{32,33}$ - were expected as correlates of pain catastrophizing.

Based on experimental studies, ${ }^{28-30}$ individual differences in trait pain catastrophizing are thought to modulate the activity of brain areas involved in selective attention. Attentional processing of salient (eg, emotional) information has been proposed to evoke dorsolateral prefrontal cortex (DLPFC) activity, ${ }^{34,35}$ supported also by the evidence on non-invasive brain stimulation applied over the DLPFC $^{36}$ If pain catastrophizing acts as an attentional modulation, then it is logical to hypothesize that dorsolateral prefrontal cortex has a role in this process. In addition, DLPFC - as a functionally heterogeneous brain area - has been implicated in the processing of painful stimuli, ${ }^{37}$ especially in processing the non-spatial sensory information - such as intensity - of painful stimuli. ${ }^{38}$ DLPFC also plays a role in pain modulation: involvement of this area in the descending modulation of pain might be exerted via attentional and other cognitive control processes. ${ }^{39}$ Therefore, it is a plausible hypothesis that activity of the DLPFC is related to pain catastrophizing.

To investigate potential mechanisms underlying pain catastrophizing, it is essential to address whether the neural correlates of pain catastrophizing are similar among pain-free controls and among chronic pain patients. It is important to note that chronic pain is considered to be associated with alterations in gray matter (GM) and in functional connectivity (FC).$^{40}$ In addition, prospective studies suggest that structural and functional connectivity in cortico-limbic circuitry predict risk for chronic pain, ${ }^{41-43}$ indicating that representation of chronic/clinical pain differ from that of acute pain. ${ }^{44,45}$ These findings corroborate our notion that neural correlates of pain catastrophizing might depend upon the presence of chronic pain.

In 2017 Malfliet et al published a systematic review on brain changes associated with cognitive and emotional factors in chronic pain. In that paper, ${ }^{46}$ pain catastrophizing was also addressed, but our review differs from that article at least in four important ways. Firstly, our starting point was pain catastrophizing (and not chronic pain as it was in Malfliet et al's review), and we aimed to reveal potential mechanisms underlying pain catastrophizing. Therefore, as a second difference, we also included studies that exclusively used a healthy control group, and thirdly, since several papers found that pain catastrophizing is an important factor in migraine-related disability, ${ }^{10,11,47,48}$ we also included studies with migraine patients (both type of studies were excluded from Malfliet et al's review). Migraine is characterized by recurrent headaches with moderate or severe pain ${ }^{49}$ and it has been proposed to be associated with similar structural and functional neural changes identified in other chronic pain populations. ${ }^{50}$ 
Therefore, our decision was to include studies with migraine patients as well. Fourthly, in our review we aimed to compare whether the neural correlates of pain catastrophizing are similar among pain-free controls and among chronic pain patients.

Summarizing and highlighting the overall goals (based on the PICO framework): the aim of the study was to answer what the neural correlates (O; Outcome) of pain catastrophizing (E; Exposure) are among chronic pain patients and/or pain-free control subjects (P; Population). We also aimed to review correlates of pain catastrophizing in pain patients compared to pain-free healthy controls $(\mathrm{C}$; Comparator). Regarding study design, we included crosssectional studies and those treatment studies which measured changes in catastrophizing along with changes in structural and/or functional neural changes (S; Study design) measured with magnetic resonance imaging (MRI) (I; diagnostic instrument).

\section{Materials and methods}

\section{Study selection}

The articles we used were selected from PubMed and the Web of Science - as they include several databases - after a thorough search. To identify the papers, we searched with the following key search terms in titles and abstracts: pain catastrophizing AND (brain activity OR neuro imaging OR imaging OR MRI OR magnetic resonance imaging) and also checked the identified references of the reviews and included studies.

The inclusion criteria were (1) articles published in English (2) between 2004 and 2016 (the last search was conducted on September 1, 2016), which used (3) the Pain Catastrophizing Scale (PCS) ${ }^{51}$ or the catastrophizing subscale of Coping Strategies Questionnaire (CSQ) ${ }^{52}$ (E: Exposure) as these are the most often used selfreport measures of pain catastrophizing. ${ }^{53}$ Other inclusion criteria were to (4) measure pain catastrophizing on a sample size of at least 12 adult participants - according to Desmond and Glover ${ }^{54}$ - diagnosed with some type of chronic pain or who were pain-free controls (P: population) and (5) report any correlation between MRI data (I: diagnostic instrument) and the catastrophizing scales (O: outcomes). We included treatment studies as well if they reported any relationship ( $r$ or $t / z$ scores reported) between changes in pain catastrophizing and any changes in GM or in connectivity and/or activity of any brain areas.
Screening, identification and eligibility (as seen on Figure 1) were conducted by two independent researchers (A.G. and Z.N.) with the initial level of agreement of $97 \%$.

With this method we found 419 articles from which 40 were duplicated. We further discarded most of the articles (333) as they were not eligible based on the title and abstract. From the remaining 46 articles, after full text analysis, an additional 26 were discarded as they were reviews, metaanalyses or did not investigate the connection between one of the pain catastrophizing scales and the neural activity measured by magnetic resonance imaging (either structural or functional [fMRI]) techniques (see in Appendix S1). Thus, 20 articles remained and were reviewed. The process, which was based on the PRISMA protocols, is shown on Figure 1.

\section{Data extraction}

Sample characteristics (size, mean age), group characteristics (whether there were chronic pain and control groups), the used questionnaires and imaging methods, confounding variables and the reported statistical thresholds (see Table 1) along with the main findings were extracted (see Table 3). In the case of task-based studies, we also recorded the type of the pain task, the modality of the painful stimulus and the body part it was given on, and also pain intensity and whether participants had to rate pain during the scan (see Table 4 for detailed information). The identification and selection of the articles were conducted by A.G. and Z.N. Data extraction from the included articles were done by A.G., Gy.K. and A.E.É. After this process, Gy.K. and N.K. thoroughly reviewed the extracted data before A.G. and E.Sz. did the risk of bias evaluation (for details, see the next section).

\section{Data synthesis}

We have focused on the data in line with our aims: we checked whether the authors compared a chronic pain group with healthy controls or just used one group. If they used multiple groups, we checked whether they reported results in both groups or only for one group. If they reported results for both groups, we checked what were the similarities and differences. With task-based studies we focused on the chosen pain task, pain intensity and the place of the stimuli.

\section{Risk of bias}

The risk of bias tool used here was based on the work of different authors. ${ }^{56-58}$ We utilized the relevant parts of widely used evaluating tools but added important (but interestingly not studied so far) new items: we 


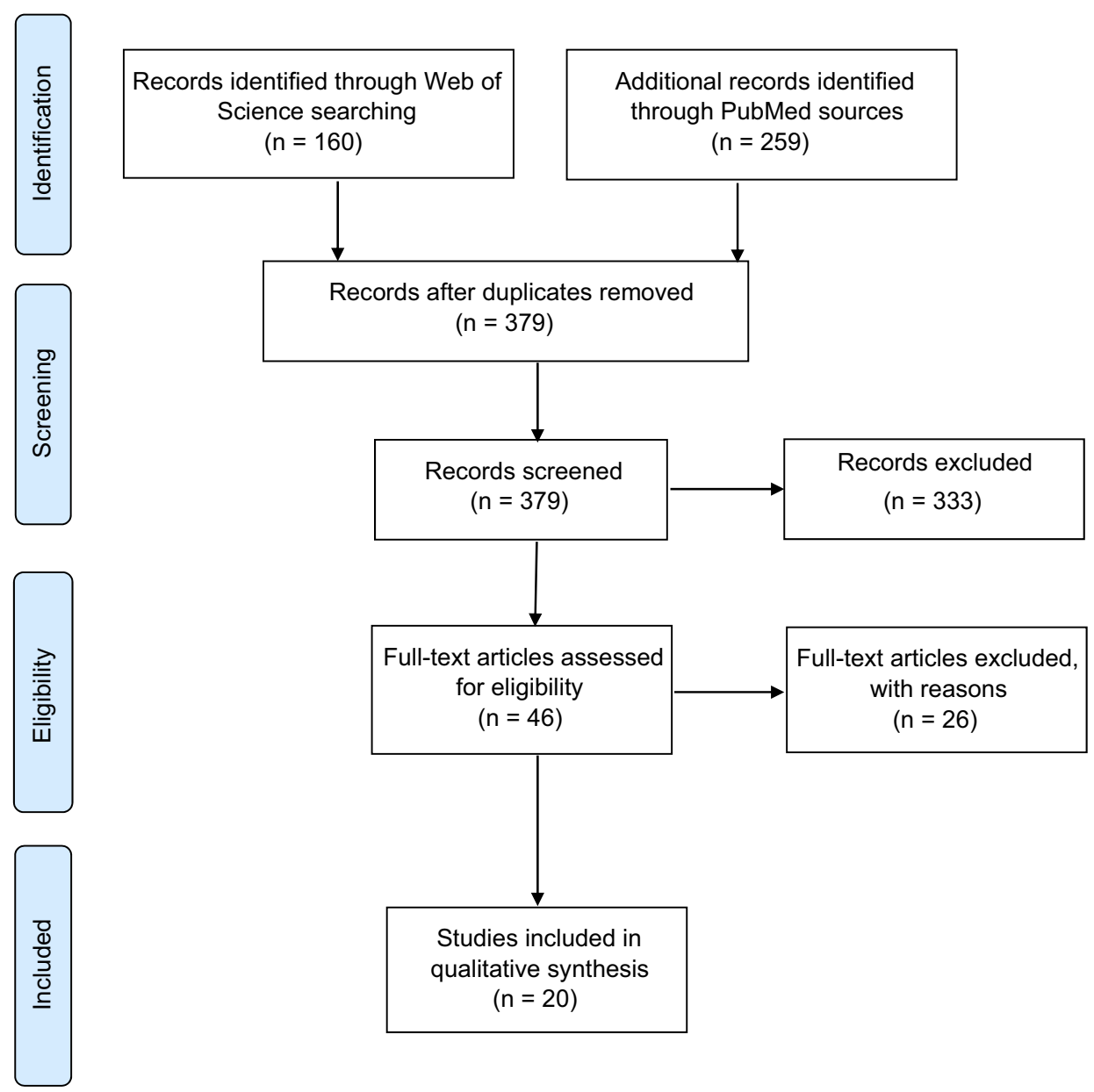

Figure I Flowchart: Selection process. Adapted from Moher D, Liberati A, Tetziaff J, Altman DG. The PRISMA Group. Preferred Reporting Items for Systematic Reviews and Meta-Analyses: the PRISMA statement. PLoS Med. 2009;6(6):el000097. ${ }^{55}$

checked for sampling method, response rate and study design, but also monitored for MRI task instructions, MRI data quality checks, used and reported thresholds (as a family wise error/false discovery rate (FWE/FDR) corrected analysis is much stronger than one with an uncorrected $p$-value) and reported outcomes (for a detailed description, see Appendix S2). We also checked for response rate and drop out rate, and although usually in smaller, cross-sectional studies it is not reported, we think that it is an important component of a study's strength. We used a simple "yes/no/ unclear/not applicable" evaluation system - based on Armijo-Olivo et al's work ${ }^{56}$ - with small exceptions (eg, at study design, percentage of response rate or the used thresholds). The more "yes" responses a study had, the stronger it was (or the lower its risk of bias was).

With this method, we obtained seven major categories (Selection bias, Study design, Detection bias, Data collection and quality check, Drop-out rate described,
Confounding variables controlled for and Reporting bias). To compute the category ratings, we used the Effective Public Health Practice Project's evaluation system, ${ }^{56}$ where categories can be marked as strong, moderate or weak. We checked the yes/no/unclear ratio in every category and marked them as weak if there were more no and unclear ratings than "yes" ratings, moderate if the yes/no/unclear ratio was equal and strong if it only had clearly reported values. Global ratings were computed as weak if there were two or more categories marked as weak, moderate with only one weak category and strong with no weak ratings.

In our system, two categories were measured with greater weight: the controlling of confounding variables and the reported threshold. With reported thresholds, we marked a study as weak if the reported threshold was uncorrected or was not reported at all. The rating was moderate if the authors controlled for multiple testing (FDR or FWE correction) but left out $z$ or $r$ or $F$ or 
$t$ scores or did not report every result set out in the aim of the study. Although threshold reporting varies greatly in neuroimaging literature and stricter standards on this reporting have only been in place in very recent years, we think that it should be taken into account while evaluating the risk of bias of former neuroimaging articles as well; therefore, we decided to apply this category.

Pain catastrophizing, depression, anxiety and fear of movements are correlated significantly; however, a number of studies suggested that pain catastrophizing is a distinct construct and has a unique effect on experienced pain intensity beyond and above depression, ${ }^{59}$ neuroticism, ${ }^{60}$ anxiety, ${ }^{9}$ and fear of pain. ${ }^{61}$ Therefore, in our systematic review we aimed to record whether confounding variables were controlled for in (MRI) studies, although we did not examine these variables separately, only checked whether they were taken into account in the studies. We rated the article as weak if there was nothing reported, moderate if the authors controlled for either task (eg, age for GM studies), group comparison or catastrophizing (eg, depression, neuroticism) relevant variables, and strong if the authors controlled for both task/group (if relevant) and catastrophizing relevant variables.

Selective reporting within studies - eg, presenting neural correlates of pain catastrophizing only in the patient group without explicitly stating whether it was tested in the control group - may bias the results or possible interpretation of the findings; therefore, we emphasized it in reporting the main findings (see Table 3). Similarly, we checked whether there were any fMRI studies in the excluded studies that used PCS, but did not use it in the analyses as a covariate.

\section{Results}

\section{Study characteristics}

From the 20 studies, we found that five studies used anatomical scan and then analyzed the data with voxelbased morphometry (VBM) or cortical thickness analysis or surface based analysis, ${ }^{62-66}$ one study used quantitative arterial spin labelling (qASL) ${ }^{67}$ and five studies used resting state fMRI measures. ${ }^{63,64,68-70}$

Ten studies used a task during the fMRI scan. ${ }^{31,71-79}$ With four exceptions ${ }^{31,66,71,75}$ the studies used the PCS to measure pain catastrophizing.

From the studies we identified, twelve used a case-control study design, ${ }^{62-65,68,69,71-73,75,78,80}$ two used cohort-analytic design, ${ }^{66,76}$ five used observational study design, ${ }^{31,67,74,77,79}$ and one study used a randomized controlled trial. ${ }^{70}$

For evaluating the neural correlates of catastrophizing, the effect of potential confounds (eg, depressive mood, anxiety or neuroticism) are suggested to be controlled for. From the 20 studies selected for our review, only four studies controlled clearly for depression and/or anxiety/neuroticism when pain catastrophizing was entered in the analysis. ${ }^{31,66,79,80}$ The included studies and their characteristics are presented and summarized in Table 1.

\section{Participants}

Apart from three studies, which only examined healthy controls, ${ }^{67,74,79}$ all studies used a chronic pain population. Six studies examined fibromyalgia patients, ${ }^{31,63,70,71,77,80}$ in two, the focus was on irritable bowel syndrome (IBS), ${ }^{62,73}$ in another two, subjects with migraine participated $^{64,78}$ and there were those who studied provoked vestibulodynia (PVD), ${ }^{65}$ osteoarthritis (OA), ${ }^{72}$ localized provoked vulvodynia (LPVD) ${ }^{68}$ or chronic pain connected to muscles, temporomandibular disorder $(\mathrm{TMD})^{69}$ or chronic low back pain. ${ }^{75,76}$ One study made a mixed group from different chronic pain patients (low back pain, myofascial pain syndrome, headache, fibromyalgia, upper body, pelvic floor). ${ }^{66}$

Mean age of chronic patient groups (with one exception $^{65}$ ) was above or exactly $30,{ }^{62,68,69,73}$ and in most cases above $40,{ }^{31,63,64,66,70-72,75-78,80}$ with three studies using chronic pain patients with a mean age of 50,51 and $62 .{ }^{66,71,72}$ In the three studies which only examined healthy controls, ${ }^{67,74,79}$ the mean age was between 25 and 35 .

Seven studies examined structural and functional correlates of pain catastrophizing on a female sample $62,63,65,68,69,71,73$ while 13 articles used mixed samples with female majority (for detailed info, see Table 1). ${ }^{31,64,66,67,70,72,74-80}$

The average sample size was 19 participants in the chronic pain groups (ranging from 11 to 58) and 15 in the control groups (between 11 and 34) in the studies we found, which is in line with studies concerning mean sample size in fMRI studies ${ }^{54,81}$

\section{Risk of bias and level of evidence}

Evaluation was made by two independent researchers (A.G. and E.Sz.) with 84\% initial agreement, consensus was achieved through either discussing the interpretation 


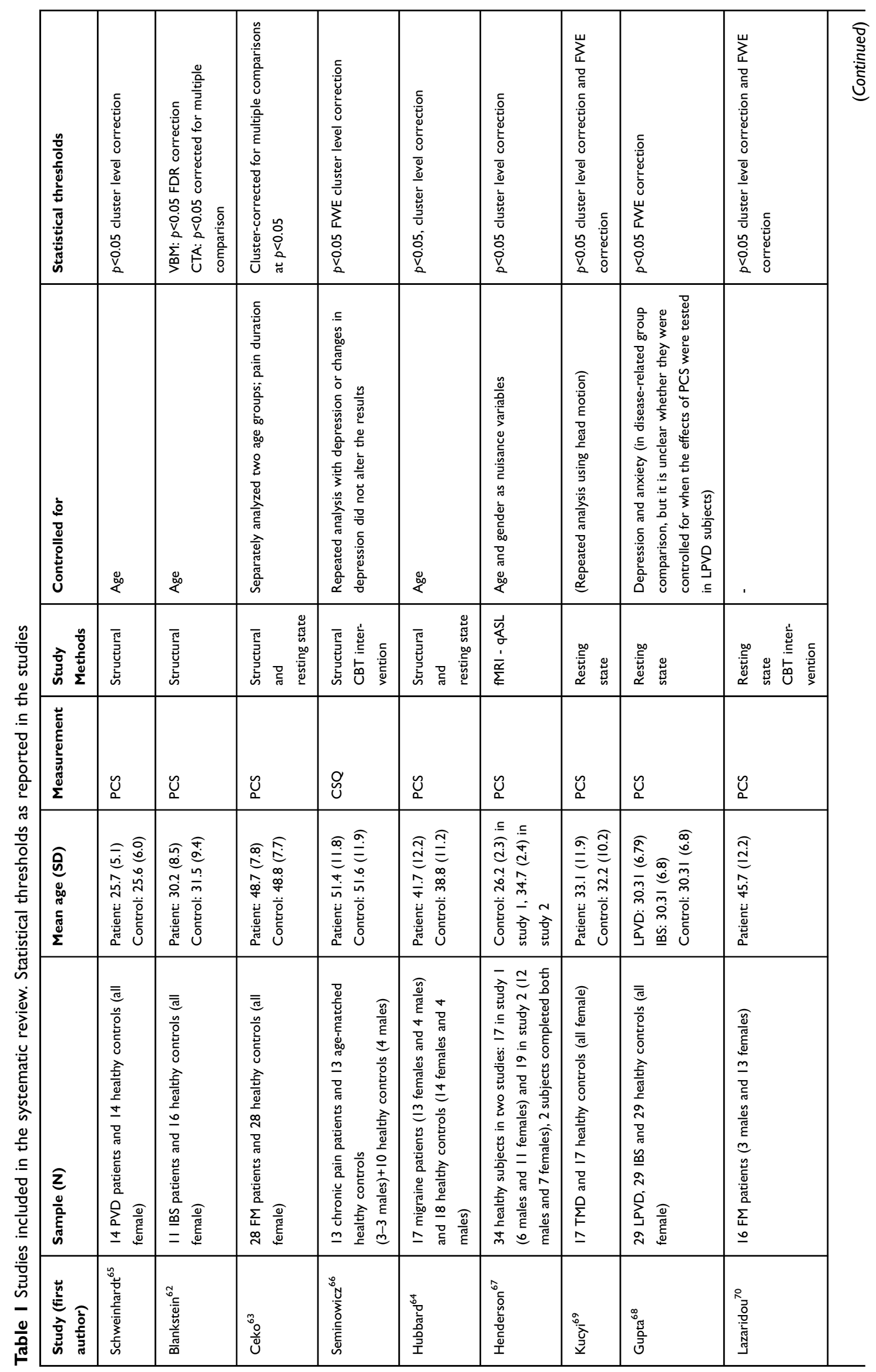




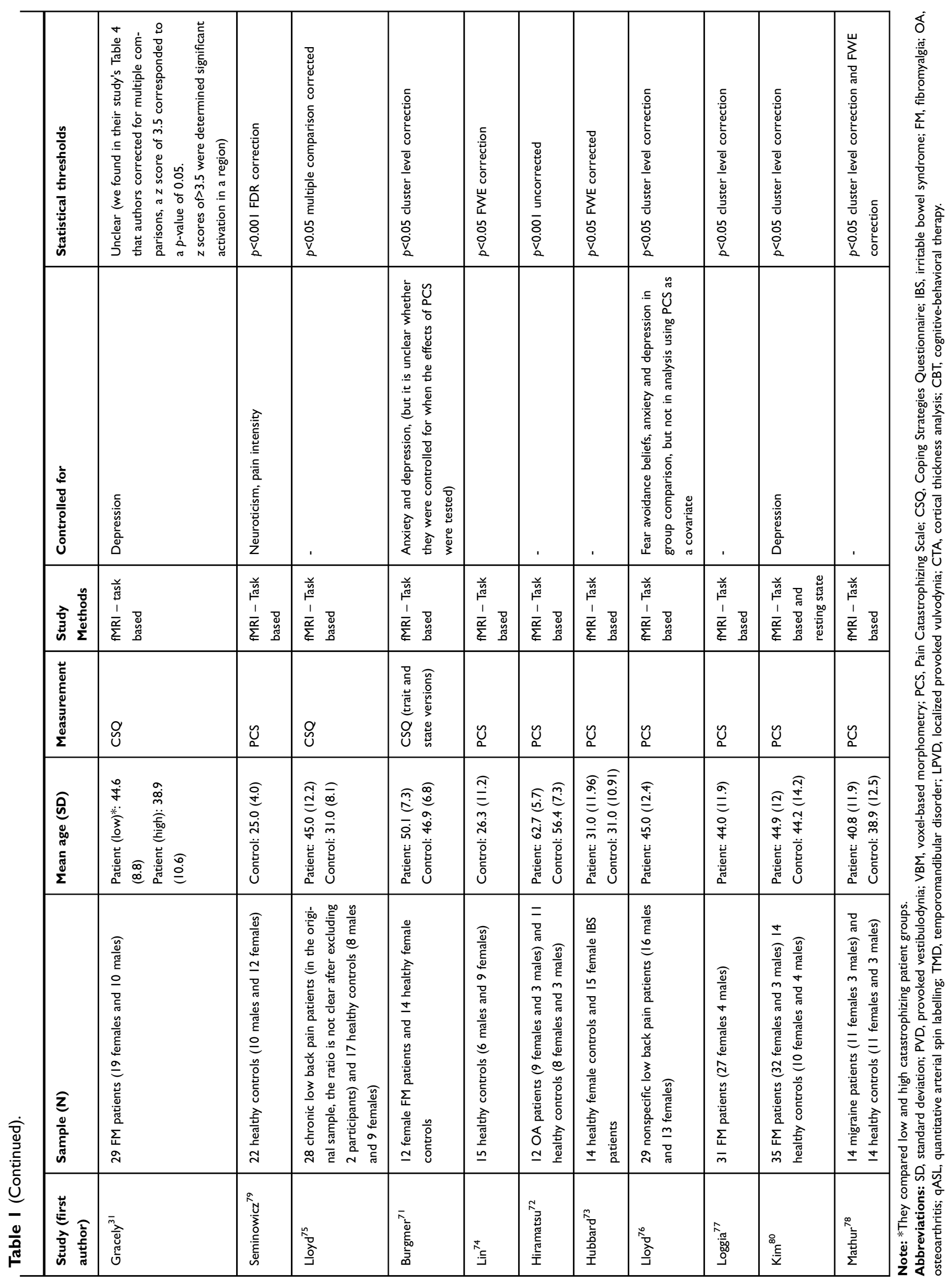


of criteria again or involving a third independent reviewer (Gy.K.).

Overall, global rating was strong only in one study, while the others were rated as moderate (nine studies) or weak (10 studies); see the detailed list in Table 2. Most of the studies lacked an adequate description of subject selection procedure and/or drop-out rates. Similarly, many studies did not account for possible confounders even in group comparisons and/or in determining the neural correlates of pain catastrophizing.

\section{Main findings}

The individual results of every article reviewed here can be found in Table 3 .

\section{Studies focusing on GM alterations}

Five studies ${ }^{62-66}$ analyzed the GM alterations in healthy and clinical subjects in correlation with pain catastrophizing (see Table 3). One of the five studies was a nonrandomized, not fully controlled study that investigated the effect of cognitive behavioral therapy (CBT) interventions only in a chronic pain sample. ${ }^{66}$ The other four studies involved a patient group with pain symptoms and a pain-free control group as well. Interestingly, in most of the studies, only female subjects were scanned and in the two mixed sample studies, females were in the majority.

Since age is associated with GM volume (GMV), taking into account its effect is important when analyzing brain structural changes. Age was controlled for in the analysis only in three studies, ${ }^{62,64,65}$ while in one study, ${ }^{63}$ participants were assigned to different groups based on their age (younger and older participants' group). One study $^{63}$ (see Table 1) used pain duration as a confounding variable in the analysis and another study ${ }^{64}$ reported a negative association between disease duration and the morphology of DLPFC in migraine.

To conclude, two studies ${ }^{62,64}$ demonstrated a significant association between pain catastrophizing and DLPFC GM morphology. Both studies found an opposite relationship between DLPFC GMV/cortical thickness and pain catastrophizing according to the study groups: in patient groups the correlation was negative, while in painfree controls, it was positive. In the migraine study, ${ }^{64}$ this opposite relationship also emerged for other structures implicated in pain perception, including the S1, anterior midcingulate cortex (aMCC), and prefrontal cortices. Similar to the two mentioned studies, a negative correlation was also found, in fibromyalgia patients, between pain catastrophizing and GM density of aINS implicated in pain perception. ${ }^{63}$ It is worth mentioning that the results of the treatment study ${ }^{66}$ also confirmed that changes in morphology of brain areas involved in pain perception (insula, ACC, S1, prefrontal cortex) and/or modulation (DLPFC) is associated with changes in pain catastrophizing. The level of evidence was moderate in four studies and was weak in one case. ${ }^{65}$ (for details, see Table 2)

\section{Functional connectivity results: studies using resting state measures}

In resting state studies, either connections of areas involved in pain perception (aINS or S1) or connections of default mode network (DMN) were tested, mainly based on theoretical consideration. According to the results, pain catastrophizing might be related to enhanced functional connectivity (FC) among areas playing a role in pain perception (S1, aINS, thalamus) $)^{63,64,70,80}$ or to enhanced connectivity within the DMN (mPFC-posterior cingulate cortex (PCC)/precuneus) ${ }^{69}$ or between the DMN and descending pain modulatory system, including the ${ }_{\text {DLPFC }}{ }^{64}$ periventricular gray $(\mathrm{PVG}) / \mathrm{PAG} .{ }^{69}$ Connectivity between the DMN and areas involved in pain perception (such as the medial thalamus) ${ }^{69}$ was also related to pain catastrophizing in patients (see Table 3).

Generally speaking, in the control group there was no association between FC of pre-defined seeds and pain catastrophizing or there was no explicit information about it. However, one study ${ }^{64}$ yielded an interesting result: resting state functional connectivity (rsFC) between PCC and DLPFC was related positively to pain catastrophizing in migraine, but the association was negative in controls.

Studies reviewed in this section did not control for any confounding variables (or at least it is unclear whether they were controlled for in the analysis using pain catastrophizing scores). The level of evidence was moderate in three cases $^{63,64,68}$ and weak in two. ${ }^{69,80}$ (for details see Table 2)

\section{Task-based activations connected to pain catastrophizing} Ten studies (see Tables 3 and 4) used a pain task to observe the effects of pain catastrophizing (although Kim et al's study ${ }^{80}$ used a pressure pain task, they were mainly interested in FC and not task evoked activation, thus we will not mention it in this part). From these 10 studies, three used only healthy controls, ${ }^{67,74,79}$ three examined exclusively a patient group $^{31,76,77}$ and four compared 


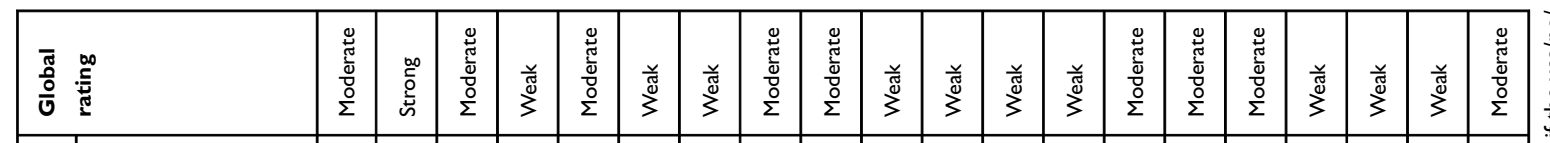

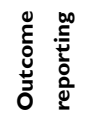

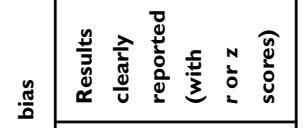

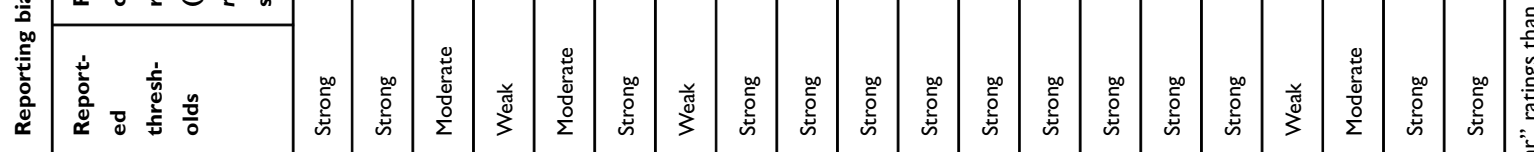

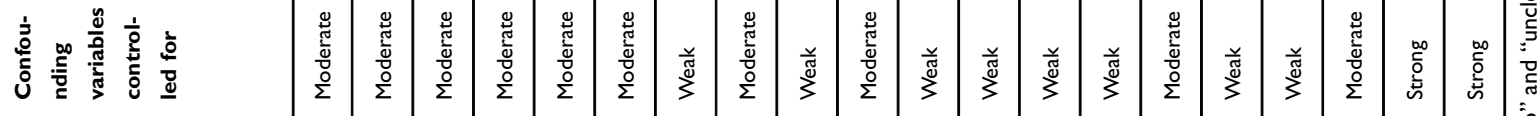

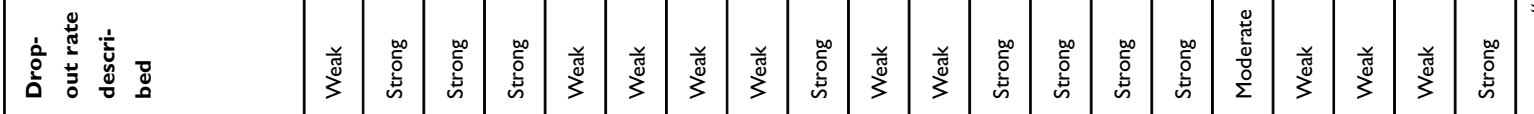

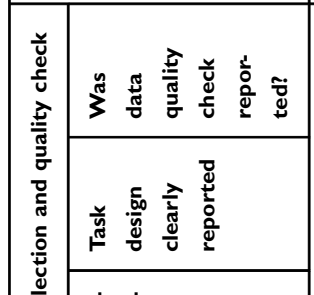

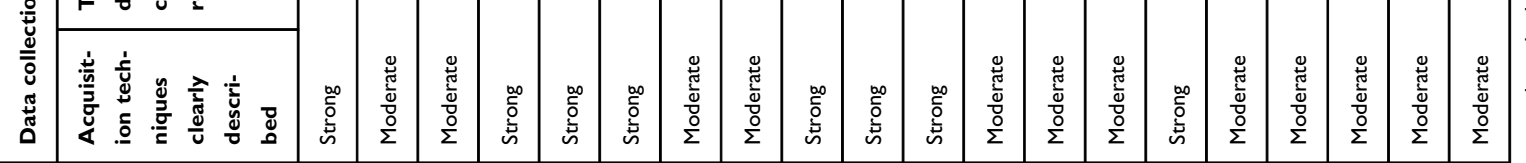

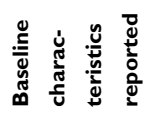

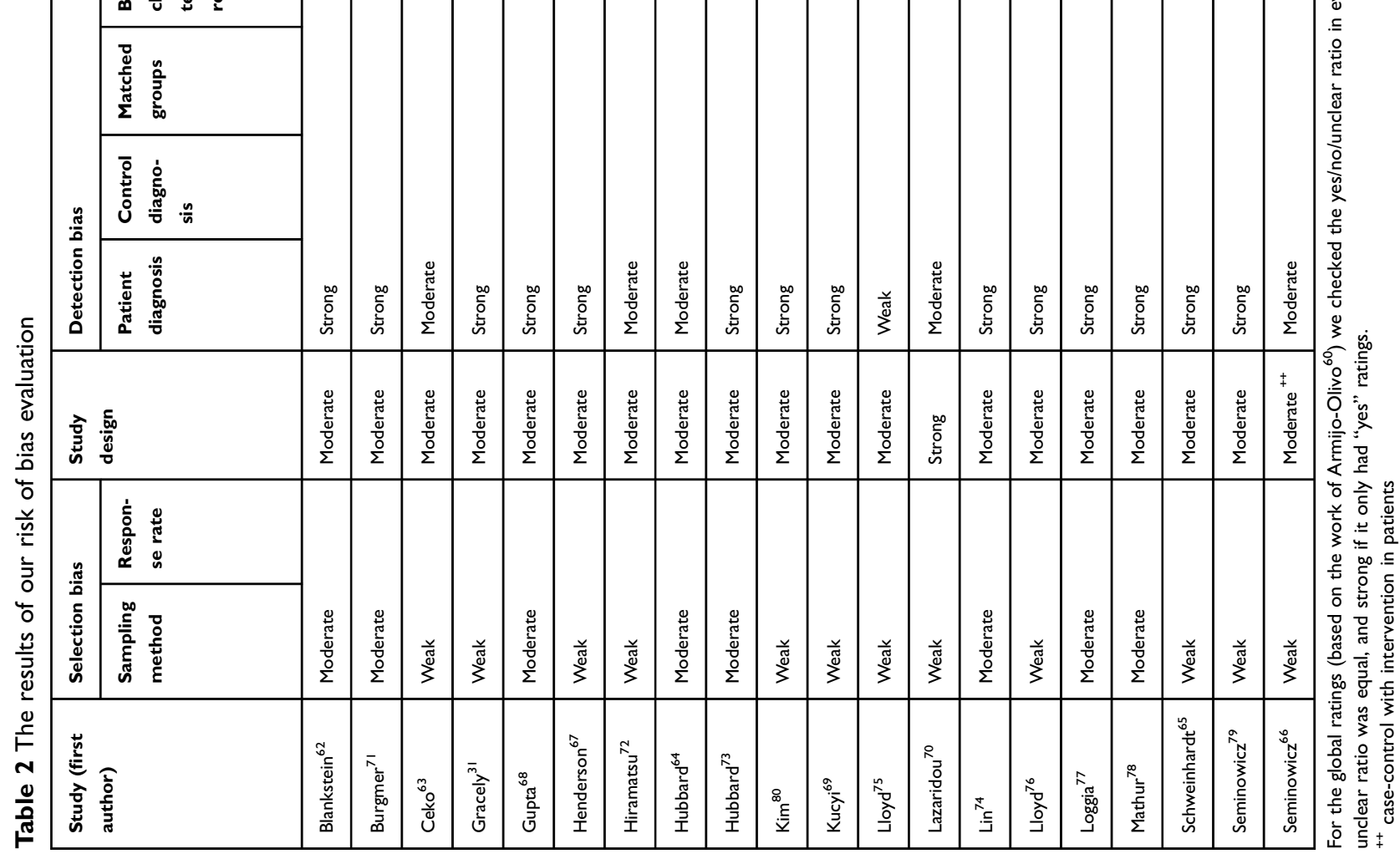




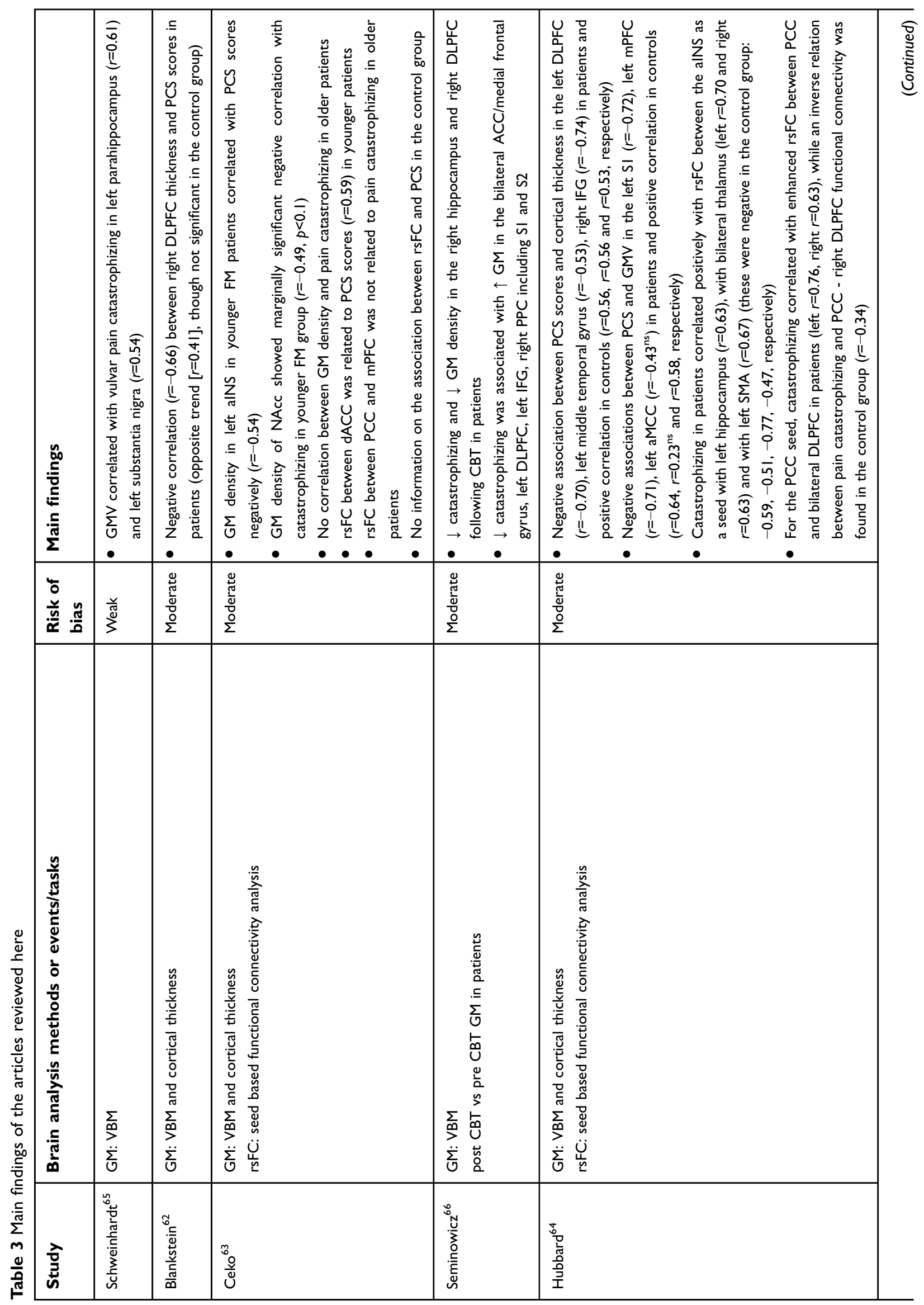




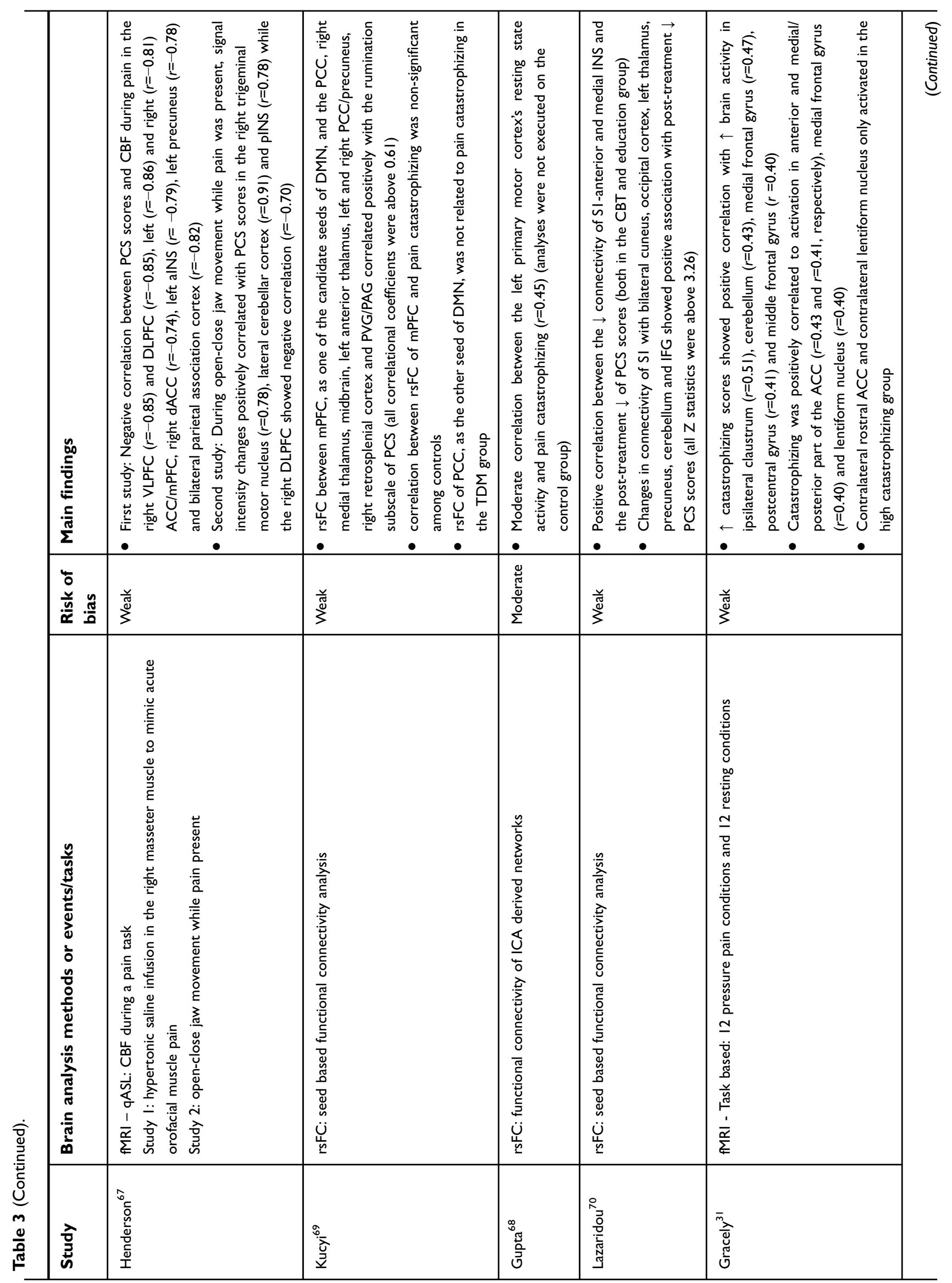




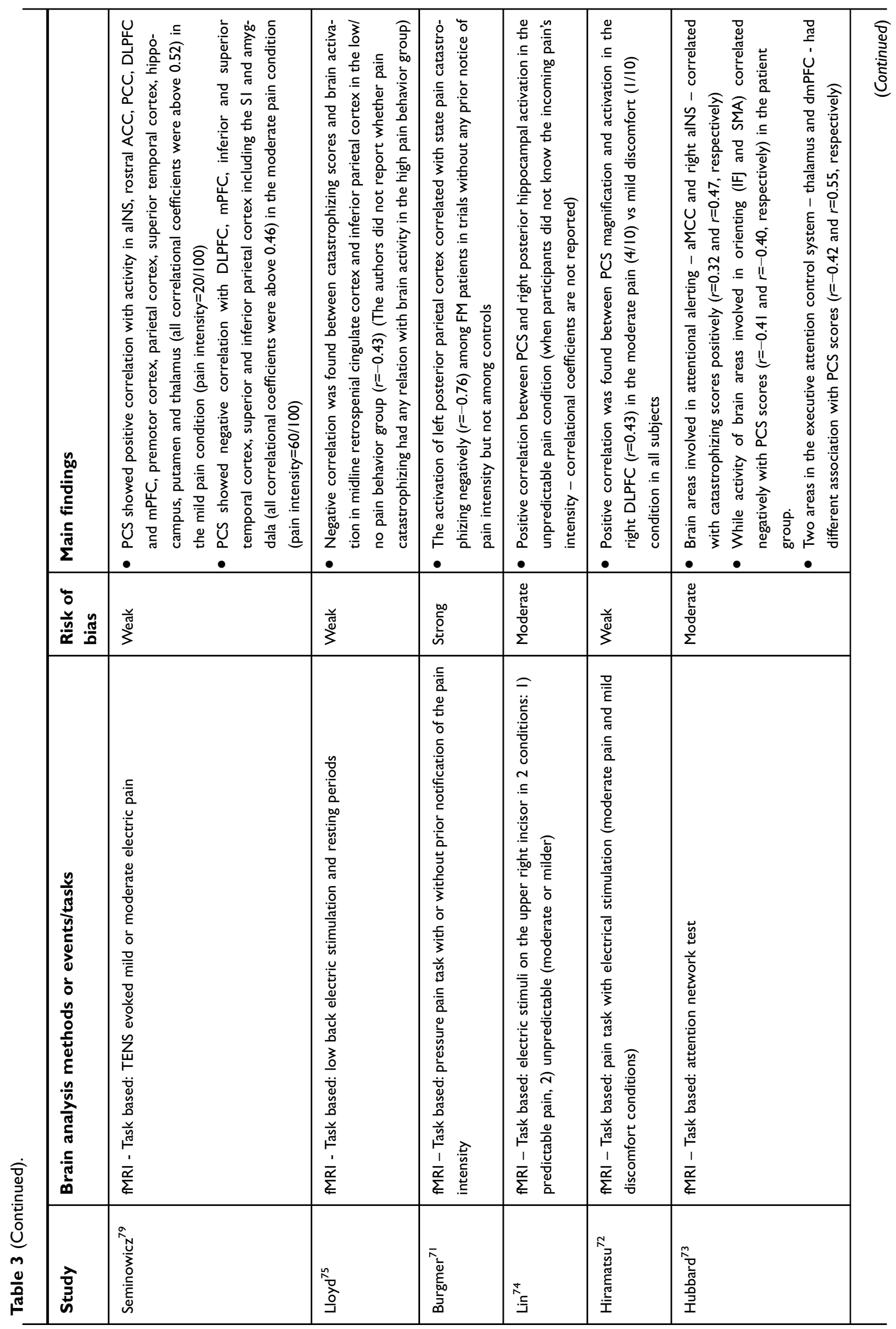




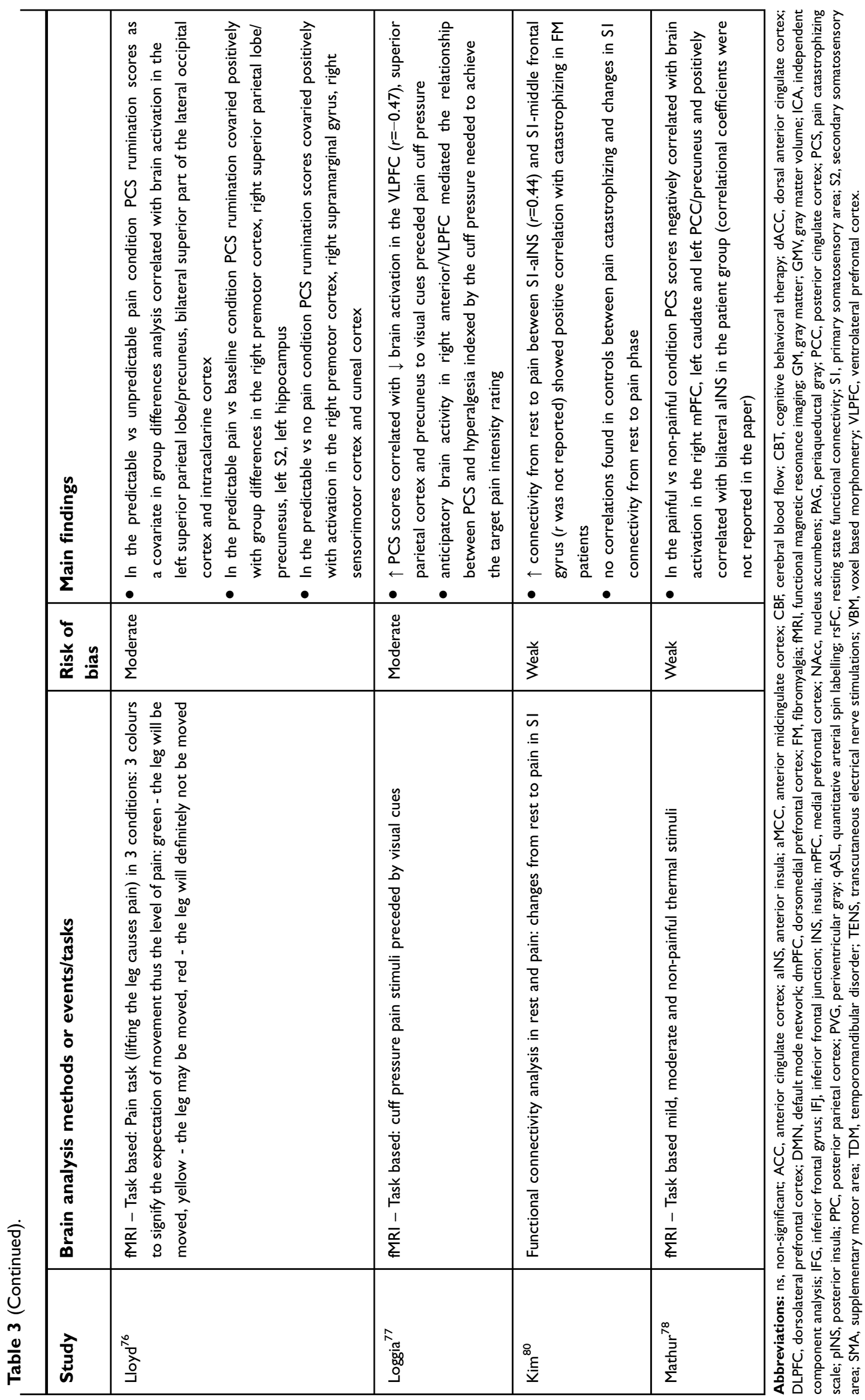




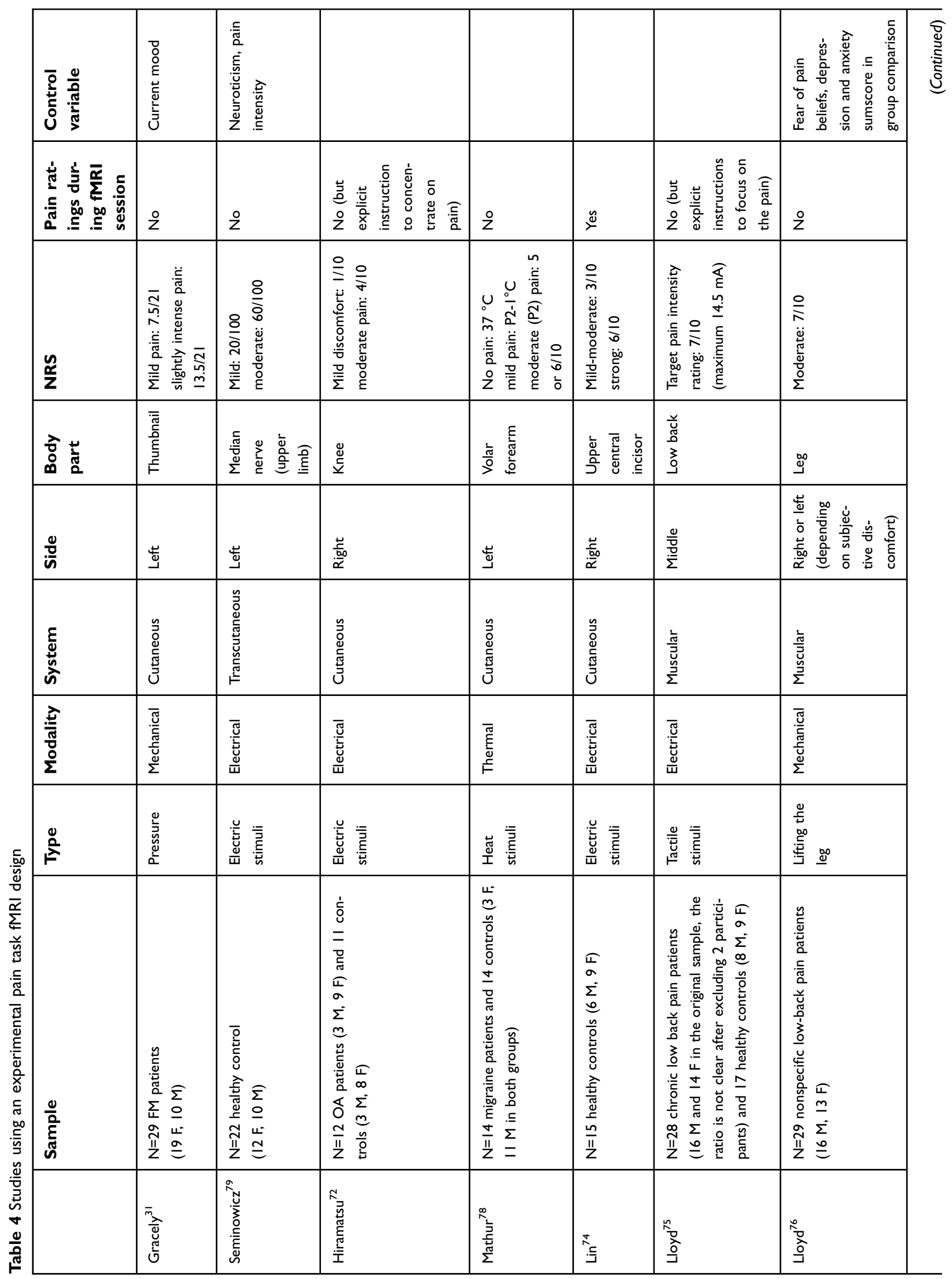




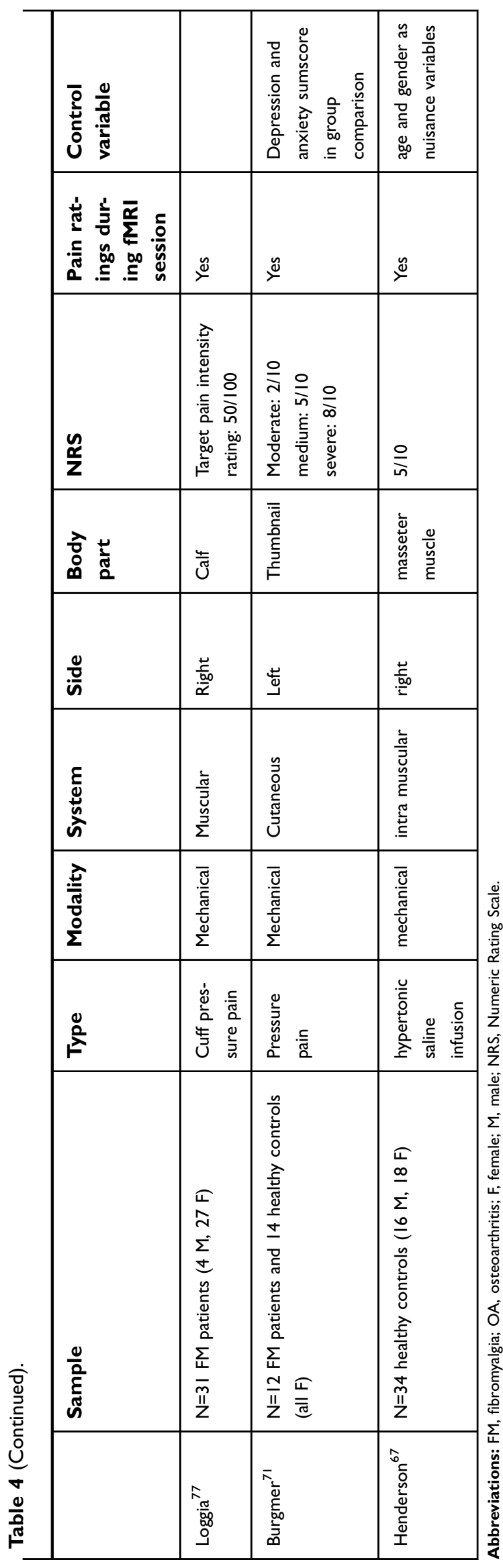

healthy controls with chronic pain patients. ${ }^{71,72,75,78}$ We also discuss Hubbard et al's work ${ }^{73}$ in this section. Although they used an attention paradigm instead of painful stimuli, they explored the effects of chronic pain and pain catastrophizing on brain activation both in controls and chronic pain patients.

Most of the studies used mechanical pain stimuli, whether it was pressure pain, ${ }^{31,71,77}$ saline infusion ${ }^{67}$ or the patient's own pain. ${ }^{76}$ Four studies used electric stimuli $^{72,74,75,79}$ and one used heat as painful stimulus. ${ }^{78}$

Although the studies used different stimuli, the most commonly associated areas with pain catastrophizing were the DLPFC, ${ }^{67,72,79}$ the insula, ${ }^{67,73,78}$ the $\mathrm{ACC},{ }^{31,70,74}$ the $\mathrm{PCC}^{78,79}$ and parts of the supplementary motor area (SMA). ${ }^{73,76,79}$

In six studies, the participants were instructed to concentrate on the painful stimuli ${ }^{71,72,74,75,77,78}$ and in three cases $^{71,74,77}$ they also had to rate the pain intensity immediately after they received the stimuli.

Comparing the studies along the pain intensity is difficult, as different authors used different scales and values to measure the intensity of the painful stimuli (for more information, see the NRS column in Table 4, where it can be seen that eg, "moderate pain" can range from 2 to 7 on a 10-point visual analog scale, depending on the study), but it cannot be ignored either. For instance, in three studies ${ }^{67,72,79}$ when the intensity of the stimulus was low $(20 / 100$ or $4 / 10)$ a positive relationship, and when the intensity of the stimulus was moderate $(5 / 10$ or $60 / 100)$ a negative relationship emerged between blood oxygenation level dependent (BOLD) activity in DLPFC and catastrophizing, which may suggest that the intensity of the stimulus may moderate the relationship between catastrophizing and brain activity.

The anticipatory phase was investigated by Loggia et $\mathrm{al}^{77}$ and by Burgmer et al. ${ }^{71}$ In these studies, the anticipatory activity in anterior/ventrolateral prefrontal cortex (VLPFC) and posterior parietal cortex was associated with pain catastrophizing, respectively.

We found that only four studies ${ }^{31,71,76,79}$ controlled for the confounding effects of other variables. Six of the studies here had low/weak level of evidence, ${ }^{31,67,72,75,79}$ three had moderate, ${ }^{74,76,77}$ and in only one case was the level of evidence strong. ${ }^{71}$

\section{Discussion}

Pain catastrophizing is consistently associated with increased pain reports; therefore, we expected that 

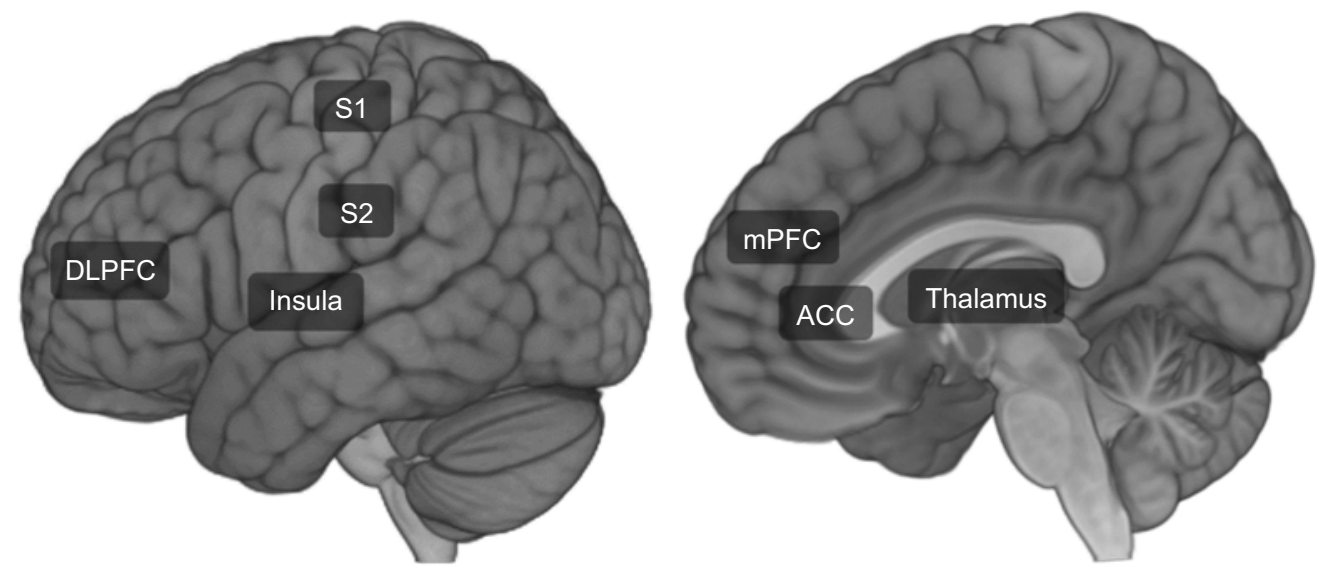

Figure 2 Most commonly reported areas in relation to pain catastrophizing in the reviewed studies.

Abbreviations: SI, primary somatosensory area; S2, secondary somatosensory area; DLPFC, dorsolateral prefrontal cortex; mPFC, medial prefrontal cortex; ACC, anterior cingulate cortex.

structural and/or functional characteristics of brain areas involved in pain perception, including the $\mathrm{S} 1$ and $\mathrm{S} 2, \mathrm{ACC}$ and MCC, insula, the PFC, the thalamus, the motor cortex, the SMA and also the brainstem, ${ }^{32,82-84}$ may be associated with increased tendency to pain catastrophizing.

Some of the areas contributing to pain perception, primarily the S1, S2, thalamus, INS and ACC, have been proposed to be involved mainly in the sensorydiscriminative and affective components of conscious pain experience. ${ }^{92}$ We found that many studies reviewed here reported a connection between pain catastrophizing and these areas (see Table 3 and Figure 2 for the main findings). GMV of these areas seems to correlate with pain catastrophizing negatively, at least among patients. For instance, higher pain catastrophizing was related to smaller GMV of ACC/MCC and S1 and GMV of aINS ${ }^{64}$ and the increase of GMV in S1 and S2 and ACC after CBT was related to decrease in pain catastrophizing ${ }^{66}$ in patients. Connectivity and task-based fMRI results suggest that increased activity of areas involved in pain processing and increased functional connectivity between them are associated with higher pain catastrophizing scores. For instance, increased connectivity between $\mathrm{S} 1$ and insula from rest to pain ${ }^{80}$ correlated pain catastrophizing positively or decrease in connectivity between S1 and insula from pre-treatment to post-treatment ${ }^{70}$ were related to decrease in pain catastrophizing. The activity of $\mathrm{S} 2^{31,76}$ also showed positive correlation to pain catastrophizing in studies using experimental pain. We found that among chronic pain patients, increased connectivity of thalamus with aINS as a seed, ${ }^{64}$ and with $\mathrm{mPFC}$ as a seed ${ }^{69}$ was associated with increased tendency to catastrophizing pain, and connectivity of the somatosensory cortex for leg with the thalamus changed with changes in pain catastrophizing after $\mathrm{CBT}^{70}{ }^{70}$ There was also a positive association between activity of aINS, ${ }^{67,73,78,79}$ and activity of $\mathrm{ACC}^{31,67,73,79}$ with pain catastrophizing in task-based studies (except in Henderson et al's study).

Based on these results, pain catastrophizing might be associated with the affective and intensity-related components of pain. Though the level of evidence in these studies was moderate to weak, it is reasonable to suggest that one of the mechanisms underlying catastrophizing cognitions is that they "make" the painful stimuli more salient and parallel, subjectively more intense, which is in line with the results of questionnaire studies. ${ }^{5-19}$

We also expected DLPFC as a key correlate of trait pain catastrophizing based on its role in attentional and pain modulatory functioning, thus contributing to the cognitive aspect of pain processing. Results of some studies reviewed here supported our expectations. GMV of DLPFC correlated with PCS scores negatively among IBS $^{62}$ and migraine patients. ${ }^{64}$ Three studies, using experimental pain, reported results on the connections between activity of DLPFC and pain catastrophizing; ${ }^{67,72,79}$ however, the results were not conclusive. Two of these studies, using an acute pain experimental design with healthy participants, only found a negative relationship between pain catastrophizing and cerebral blood flow (CBF) in right DLPFC during acute moderate pain $(5 / 10)^{67}$ and BOLD activity of bilateral DLPFC during acute moderate pain $(60 / 100) .{ }^{79}$ When the pain was only mild (20/100) in that latter study, the relationship was the opposite: positive correlation with pain catastrophizing, ${ }^{79}$ similarly to 
a mixed sample (patients + controls) study, in which participants were investigated during moderate pain (4/10) vs mild discomfort (1/10). ${ }^{72}$ Direct comparison of these results is not easy since labelling pain intensity and the used numeric rating scales varied from study to study (see Table 4). In addition, methodological quality of fMRI studies that provided an association between the activation of DLPFC in task-based studies and pain catastrophizing was weak, thus it is hard to come to a definite conclusion on the potential role of DPLFC in catastrophizing.

We also aimed to review the neural correlates of pain catastrophizing in pain patients and pain-free controls. Concerning DLPFC, two studies ${ }^{62,64}$ found opposite association between GM density of DLPFC and pain catastrophizing in patients and healthy controls. They found that decreased DLPFC GM density is associated with increased tendency to catastrophize pain in patients (IBS and migraine patients). As the DLPFC is part of the descending pain modulation system, which can modify the pain experience, the decreased amount of DLPFC GM might explain the increased amount of pain perception in chronic pain. ${ }^{84,85}$ However, in the control groups a positive association was found between GMV density of DLPFC and pain catastrophizing. Those who had higher pain catastrophizing scores had higher GM density in DLPFC, suggesting different mechanisms underlying pain catastrophizing among controls and pain patients. This idea is supported by other results: one study ${ }^{64}$ in our review found that relationship between catastrophizing and GMV of other brain regions - such as inferior frontal gyrus (IFG), S1, aMCC, mPFC - also had an opposite relationship in controls and in (migraine) patients. However, it is not clear whether these results can be generalizable to other pain conditions compared to controls.

Differences between healthy subjects and pain patients might suggest that structural and functional brain alterations related to pain catastrophizing may depend on prior and/or relatively stable/constant pain experience. It is worth noting that pain conditions (such as fibromyalgia, IBS, migraine, TMD, and low back pain) investigated in studies selected for our review may differ in some specific structural and functional brain alterations, but may share similar alterations within regions involved in pain processing and pain modulation, such as the INS, ACC, PFC (for a review see the work of Davis ${ }^{85}$ or Bushnell ${ }^{86}$ ). Chronic pain related neural reorganization in GM, white matter or brain connectivity is hypothesized to be accompanied by a shift in the salience of the pain. ${ }^{87}$ When chronic pain develops, painful stimuli are no longer just external threats but an inherent part of the everyday experience of patients. According to our review, regardless of the type of the chronic pain, neural correlates and mechanisms of pain catastrophizing might be similar across disorders: catastrophizing might be associated with enhanced intensity and affective processing, along with increased attentional processes towards painful stimuli and/or weakened modulation of pain.

\section{Limitations and future recommendations}

One major limitation of the reviewed studies is that most of the participants were females. One explanation to this might be that the prevalence of chronic pain is slightly higher in women than in men. ${ }^{88}$ Another limitation would be the different age the reviewed studies reported. In most cases, the mean age of the patient group was well above 30 . While in most of the studies, the authors used an age matched control group, generally the healthy participants are younger.

Although the average number of participants was above twelve in line with the findings of Desmond ${ }^{54}$ and David ${ }^{81}$, they also say that for reliable results, one needs at least twice this many in each group. In addition, quality of the reviewed studies was moderate to weak. This might be one of the reasons we could not find similar results in the reviewed studies.

It has been proposed that GM alterations in chronic pain are at least partially due to chronic pain itself, ${ }^{87,89,90}$ therefore duration of the disease should be controlled for; however, only one study controlled for pain duration, ${ }^{63}$ and many studies did not check confounding variables at all in the analyses.

It is also worth mentioning that in some studies that compared two groups - pain-free control group vs patient group - the results on pain catastrophizing of the healthy participants are not explicitly published, which makes the comparison nearly impossible. Our intention to compare neural correlates of pain catastrophizing in patient and in pain free participants sheds light on another shortcoming of pain catastrophizing studies: PCS asks about general painrelated thoughts which, for a chronic pain patient, might be the actual clinical pain, while a healthy participant might recall a distant memory of a painful event. In relation to this, studies reviewed here did not evaluate the interaction 
between the actual spontaneous pain in chronic pain patients and the acute painful stimuli that were administered. In addition, generalizability of findings on neural correlates of pain catastrophizing among chronic pain patients still remained in question, since only some types of chronic pain were explored in the studies we found. More than half of the studies (10 from 16 involving chronic pain patient samples) observed fibromyalgia, irritable bowel syndrome and migraine, while in the rest mainly muscolosceletal pain was addressed.

Only one study in our review evaluated the effect of current (state) pain catastrophic thoughts which emerged during experimental pain. ${ }^{71}$ Thus we have no information about how state and trait pain catastrophizing might interact in processing or anticipating painful stimuli.

In this review, we saw that fMRI studies using a pain task to observe connection between pain catastrophizing and brain activation applied different scales to rate pain intensity. In addition, labels (eg, "moderate") for pain intensity varied from study to study, therefore direct comparison of these results was challenged.

Besides pain perception, anticipatory processes could be interesting in relation to pain catastrophizing, but only two studies investigated this relationship. ${ }^{71,77}$ Cues signalling subsequent painful stimuli may differ in their level of pain predictability. We found only two studies that tested predictable and unpredictable painful stimuli, but they did not analyze brain response to cues related to predictable and unpredictable pain in relation to pain catastrophizing. ${ }^{74,76}$

Another limitation could be our risk of bias tool. Although we based our tool on frequently used and accepted ones, we added some MRI specific items such as statistical threshold corrected for multiple testing.

For better understanding of the effect of pain catastrophizing, direct manipulation of pain catastrophic thoughts, while anticipating and perceiving pain, could be a useful way to investigate the mechanisms underlying catastrophizing. Difficulties in attentional disengagement from pain-related information have been hypothesized as a key process underlying pain catastrophizing. ${ }^{27-30}$ However, the design of fMRI studies with pain tasks we reviewed here does not allow any conclusions to be drawn about that. If catastrophizing can be conceptualized as an expectation, ${ }^{91}$ studies on anticipation of pain would deserve more attention in relation to pain catastrophizing. In addition, induction of catastrophic thoughts during anticipation or perception of painful stimuli could help to explore whether the correlates of catastrophizing are similar or different across pain-free and chronic pain samples. The use of a unified rating scale is advised for future catastrophizing and pain-task studies for easier comparison. It is important to mention that the DLPFC - identified as a hypothesized key area in our introduction - is not an anatomical region, but rather a functional one, thus a meta-analytic approach would identify more precisely which particular areas of DLPFC (or any other regions) are related to pain catastrophizing if more studies with strong evidence would be available.

\section{Conclusions}

Based on the results reviewed here, we can conclude that pain catastrophizing might be related to salience detection, pain processing, and top-down attentional processes. We found this association across a range of brain imaging modalities; thus, our review highlights the complex and moderate to weak association between pain catastrophizing and the activity or morphology/connectivity of brain areas relating to these processes.

Our results also point out that these processes in relation to pain catastrophizing are more pronounced in chronic pain patients. In addition, some of the results reviewed here suggest different correlates (and perhaps mechanisms) underlying pain catastrophizing among controls and pain patients. However, it is not obvious whether the presence or experience of chronic pain is associated with structural and functional changes or instead methodological issues (namely that the measured pain catastrophizing is related to a distant memory, as in healthy controls, or a current disturbing painful disorder, as in the chronic pain patients) are responsible for these differences. To improve prevention and treatment of painful conditions, longitudinal studies of healthy subjects with high pain catastrophizing would be required to understand which pain catastrophizing related brain mechanisms contribute to the transformation of acute pain states into chronic pain syndromes.

\section{Abbreviation list}

$\mathrm{ACC}$, anterior cingulate cortex; aINS, anterior insula; aMCC, anterior midcingulate cortex; BOLD, blood oxygenation level dependent; CBF, cerebral blood flow; CBT, cognitive behavioral therapy; CEN, cognitive executive network; CSQ, Coping Strategies Questionnaire; dACC, dorsal anterior cingulate cortex; DLPFC, dorsolateral prefrontal cortex; DMN, default mode network; dmPFC, dorsomedial prefrontal cortex; FC, functional connectivity; FDR, false discovery rate; FM, fibromyalgia; (f)MRI, 
(functional) magnetic resonance imaging; FWE, family wise error; GMV, gray matter volume; IBS, irritable bowel syndrome; IFG, inferior frontal gyrus; LPVD, localized provoked vulvodynia; $\mathrm{mPFC}$, medial prefrontal cortex; NAcc, nucleus accumbens; NFR, nociceptive flexion reflex; OA, osteoarthritis; PAG, periaqueductal gray; PCC, posterior cingulate cortex; PCS, Pain Catastrophizing Scale; pgACC, pregenual anterior cingulate cortex; pINS, posterior insula; PPC, posterior parietal cortex; PVD, provoked vestibulodynia; PVG, periventricular gray; qASL, quantitative arterial spin labeling; rsFC, resting state functional connectivity; S1, primary somatosensory area; S2, secondary somatosensory area; SMA, supplementary motor area; TMD, temporomandibular disorder; TMJD, temporomandibular muscle and joint disorders; VBM, voxel based morphometry; VLPFC, ventrolateral prefrontal cortex; vmPFC, ventromedial prefrontal cortex.

\section{Acknowledgments}

The author(s) disclosed receipt of the following financial support for the research, authorship, and/or publication of this article: the study was supported by the MTA-SE-NAP B Genetic Brain Imaging Migraine Research Group, Hungarian Academy of Sciences, Semmelweis University (Grant No. KTIA_NAP_132-2015-0001); Hungarian Brain Research Programe (Grant No. 2017-1.2.1-NKP-2017-00002) and the Hungarian Academy of Sciences (MTA-SE Neuropsychopharmacology and Neurochemistry Research Group). Andrea Edit Edes was supported by the ÚNKP-17-3-IV-SE-3 New National Excellence Program of the Ministry of Human Capacities. Edina Szabo was supported by the ÚNKP-17-3-III-ELTE-346 New National Excellence Program of the Ministry of Human Capacities. Natalia Kocsel was supported by the ÚNKP-18-3-III-ELTE-495 New National Excellence Program of the Ministry of Human Capacities. The preparation of this article was supported by the Hungarian National Research, Development and Innovation Office (Grant No. FK128614).

\section{Disclosure}

The authors declare that this work was conducted in the absence of any commercial or financial relationships that could be construed as a potential conflict of interest.

\section{References}

1. Sullivan MJ, Thorn B, Haythornthwaite JA, et al. Theoretical perspectives on the relation between catastrophizing and pain. Clin J Pain. 2001;17(1):52-64.

2. France CR, Keefe FJ, Emery CF, et al. Laboratory pain perception and clinical pain in post-menopausal women and age-matched men with osteoarthritis: relationship to pain coping and hormonal status. Pain. 2004;112(3):274-281. doi:10.1016/j.pain.2004.09.007

3. Pallegama RW, Ariyasinghe S, Perera ED, Treede R-D. Influence of catastrophizing and personality traits on recalled ratings of acute pain experience in healthy young adults. Pain Med. 2017;18 (1):49-60. doi:10.1093/pm/pnw123

4. Noel M, Rabbitts JA, Tai GG, Palermo TM. Remembering pain after surgery: a longitudinal examination of the role of pain catastrophizing in children's and parents' recall. Pain. 2015;156 (5):800-808. doi:10.1097/j.pain.0000000000000102

5. Edwards RR, Bingham CO, Bathon J, Haythornthwaite JA. Catastrophizing and pain in arthritis, fibromyalgia, and other rheumatic diseases. Arthritis Rheum. 2006;55(2):325-332. doi:10.1002/art.21865

6. George SZ, Calley D, Valencia C, Beneciuk JM. Clinical investigation of pain-related fear and pain catastrophizing for patients with low back pain. Clin J Pain. 2011;27(2):108-115. doi:10.1097/ AJP.0b013e3181f21414

7. Buenaver LF, Edwards RR, Smith MT, Gramling SE, Haythornthwaite JA. Catastrophizing and pain-coping in young adults: associations with depressive symptoms and headache pain. J Pain. 2008;9(4):311-319. doi:10.1016/j.jpain.2007.11.005

8. Feinstein AB, Sturgeon JA, Darnall BD, et al. The effect of pain catastrophizing on outcomes: a developmental perspective across children, adolescents, and young adults with chronic pain. $J$ Pain. 2017;18(2):144-154. doi:10.1016/j.jpain.2016.10.009

9. Tran ST, Jastrowski Mano KE, Hainsworth KR, et al. Distinct influences of anxiety and pain catastrophizing on functional outcomes in children and adolescents with chronic pain. J Pediatr Psychol. 2015;40(8):744-755. doi:10.1093/jpepsy/ jsv029

10. Bond DS, Buse DC, Lipton RB, et al. Clinical pain catastrophizing in women with migraine and obesity. Headache J Head Face Pain. 2015;55(7):923-933. doi:10.1111/head.12597

11. Holroyd KA, Drew JB, Cottrell CK, Romanek KM, Heh V. Impaired functioning and quality of life in severe migraine: the role of catastrophizing and associated symptoms. Cephalalgia Int J Headache. 2007;27 (10):1156-1165. doi:10.1111/j.1468-2982.2007.01420.x

12. Besen E, Gaines B, Linton SJ, Shaw WS. The role of pain catastrophizing as a mediator in the work disability process following acute low back pain. J Appl Biobehav Res. 2017;22(1):e12085. doi:10.1111/ jabr. 12085

13. Martel MO, Jamison RN, Wasan AD, Edwards RR. The association between catastrophizing and craving in patients with chronic pain prescribed opioid therapy: a preliminary analysis. Pain Med. 2014;15(10):1757-1764. doi:10.1111/pme.12416

14. Theunissen M, Peters ML, Bruce J, Gramke H-F, Marcus MA. Preoperative anxiety and catastrophizing: a systematic review and meta-analysis of the association with chronic postsurgical pain. Clin $J$ Pain. 2012;28(9):819-841. doi:10.1097/ AJP.0b013e 31824549d6

15. Velly AM, Look JO, Carlson C, et al. The effect of catastrophizing and depression on chronic pain - a prospective cohort study of temporomandibular muscle and joint pain disorders. Pain. 2011;152 (10):2377-2383. doi:10.1016/j.pain.2011.07.004

16. Mankovsky T, Lynch M, Clark A, Sawynok J, Sullivan MJ. Pain catastrophizing predicts poor response to topical analgesics in patients with neuropathic pain. Pain Res Manag. 2012;17(1):10-14. 
17. Sullivan MJ, Lynch ME, Clark AJ, Mankovsky T, Sawynok J. Catastrophizing and treatment outcome: differential impact on response to placebo and active treatment outcome. Contemp Hypn. 2008;25(3-4):129-140. doi:10.1002/ch.365

18. Scott EL, Kroenke K, Wu J, Yu Z. Beneficial effects of improvement in depression, pain catastrophizing, and anxiety on pain outcomes: a 12-month longitudinal analysis. J Pain. 2016;17 (2):215-222. doi:10.1016/j.jpain.2015.10.011

19. France CR, France JL, al'Absi M, Ring C, McIntyre D. Catastrophizing is related to pain ratings, but not nociceptive flexion reflex threshold. Pain. 2002;99(3):459-463.

20. Kunz M, Hennig J, Karmann AJ, Lautenbacher S. Relationship of 5-HTTLPR polymorphism with various factors of pain processing: subjective experience, motor responsiveness and catastrophizing. Trullas R, ed. PLoS One; 2016; 11(4):e0153089. doi:10.1371/journal.pone. 0153089

21. Sterling M, Hodkinson E, Pettiford C, Souvlis T, Curatolo M. Psychologic factors are related to some sensory pain thresholds but not nociceptive flexion reflex threshold in chronic whiplash. Clin J Pain. 2008;24(2):124-130. doi:10.1097/AJP.0b013e3 1815 ca293

22. Vase L, Egsgaard LL, Nikolajsen L, Svensson P, Jensen TS, ArendtNielsen L. Pain catastrophizing and cortical responses in amputees with varying levels of phantom limb pain: a high-density EEG brain-mapping study. Exp Brain Res. 2012;218(3):407-417. doi:10.1007/s00221-012-3027-6

23. Rhudy JL, France CR. Defining the nociceptive flexion reflex (NFR) threshold in human participants: A comparison of different scoring criteria. Pain. 2007;128(3):244-253. doi:10.1016/j. pain.2006.09.024

24. Rhudy JL, France CR, Bartley EJ, Williams AE, McCabe KM, Russell JL. Does pain catastrophizing moderate the relationship between spinal nociceptive processes and pain sensitivity? J Pain. 2009;10(8):860-869. doi:10.1016/j.jpain.2009.02.005

25. Rhudy JL, Martin SL, Terry EL, et al. Pain catastrophizing is related to temporal summation of pain but not temporal summation of the nociceptive flexion reflex. Pain. 2011;152(4):794-801. doi:10.1016/j.pain.2010.12.041

26. Rice DA, Parker RS, Lewis GN, Kluger MT, McNair PJ. Pain catastrophizing is not associated with spinal nociceptive processing in people with chronic widespread pain. Clin J Pain. 2017;33 (9):804-810. doi:10.1097/AJP.0000000000000464

27. Fashler S, Katz J. More than meets the eye: visual attention biases in individuals reporting chronic pain. J Pain Res. 2014;557. doi: $10.2147 / J P R . S 67431$

28. Van Damme S, Crombez G, Eccleston C. The anticipation of pain modulates spatial attention: evidence for pain-specificity in high-pain catastrophizers. Pain. 2004;111(3):392-399. doi:10.1016/j.pain.2004.07.022

29. Vervoort T, Caes L, Crombez G, et al. Parental catastrophizing about children's pain and selective attention to varying levels of facial expression of pain in children: A dot-probe study. Pain. 2011;152(8):1751-1757. doi:10.1016/j.pain.2011.03.015

30. Zheng C, Wang J-Y, Luo F. Painful faces-induced attentional blink modulated by top-down and bottom-up mechanisms. Front Psychol. 2015;6. doi:10.3389/fpsyg.2015.00695.

31. Gracely RH. Pain catastrophizing and neural responses to pain among persons with fibromyalgia. Brain. 2004;127(4):835-843. doi:10.1093/brain/awh098

32. Apkarian AV, Bushnell MC, Treede R-D, Zubieta J-K. Human brain mechanisms of pain perception and regulation in health and disease. Eur J Pain. 2005;9(4):463. doi:10.1016/j.ejpain.2004.11.001

33. Tracey I, Mantyh PW. The cerebral signature for pain perception and its modulation. Neuron. 2007;55(3):377-391. doi:10.1016/j. neuron.2007.07.012
34. Bishop SJ. Trait anxiety and impoverished prefrontal control of attention. Nat Neurosci. 2009;12(1):92-98. doi:10.1038/nn.22 42

35. MacDonald AW, Cohen JD, Stenger VA, Carter CS. Dissociating the role of the dorsolateral prefrontal and anterior cingulate cortex in cognitive control. Science. 2000;288(5472):1835-1838.

36. Mondino M, Thiffault F, Fecteau S. Does non-invasive brain stimulation applied over the dorsolateral prefrontal cortex non-specifically influence mood and emotional processing in healthy individuals? Front Cell Neurosci. 2015;9. doi:10.3389/ fncel.2015.00399.

37. Duerden EG, Albanese M-C. Localization of pain-related brain activation: A meta-analysis of neuroimaging data. Hum Brain Mapp. 2013;34(1):109-149. doi:10.1002/hbm.21416

38. Oshiro Y, Quevedo AS, McHaffie JG, Kraft RA, Coghill RC. Brain mechanisms supporting discrimination of sensory features of pain: a new model. J Neurosci. 2009;29(47):14924-14931. doi:10.1523/ JNEUROSCI.5538-08.2009

39. Seminowicz DA, Moayedi M. The dorsolateral prefrontal cortex in acute and chronic pain. $J$ Pain. 2017;18(9):1027-1035. doi:10.1016/j.jpain.2017.03.008

40. Farmer MA, Baliki MN, Apkarian AV. A dynamic network perspective of chronic pain. Neurosci Lett. 2012;520(2):197-203. doi:10.1016/j.neulet.2012.05.001

41. Baliki MN, Petre B, Torbey S, et al. Corticostriatal functional connectivity predicts transition to chronic back pain. Nat Neurosci. 2012;15(8):1117-1119. doi:10.1038/nn.3153

42. Mansour AR, Baliki MN, Huang L, et al. Brain white matter structural properties predict transition to chronic pain. Pain. 2013;154(10):2160-2168. doi:10.1016/j.pain.2013.06.044

43. Vachon-Presseau E, Tétreault P, Petre B, et al. Corticolimbic anatomical characteristics predetermine risk for chronic pain. Brain. 2016;139(7):1958-1970. doi:10.1093/brain/aww100

44. Baliki MN, Chialvo DR, Geha PY, et al. Chronic pain and the emotional brain: specific brain activity associated with spontaneous fluctuations of intensity of chronic back pain. $J$ Neurosci. 2006;26 (47):12165-12173. doi:10.1523/JNEUROSCI.3576-06.2006

45. Hashmi JA, Baliki MN, Huang L, et al. Shape shifting pain: chronification of back pain shifts brain representation from nociceptive to emotional circuits. Brain. 2013;136(9):2751-2768. doi:10.1093/brain/awt211

46. Malfliet A, Coppieters I, Van Wilgen P, et al. Brain changes associated with cognitive and emotional factors in chronic pain: A systematic review. Eur $J$ Pain. 2017;21(5):769-786. doi:10.1002/ejp. 1003

47. Galioto R, O'Leary KC, Thomas JG, et al. Lower inhibitory control interacts with greater pain catastrophizing to predict greater pain intensity in women with migraine and overweight/obesity. J Headache Pain. 2017;18:1. doi:10.1186/s10194-017-0748-8

48. Seng EK, Buse DC, Klepper JE, et al. Psychological factors associated with chronic migraine and severe migraine-related disability: an observational study in a tertiary headache center: headache. Headache J Head Face Pain. 2017;57(4):593-604. doi:10.1111/head.13021

49. Silberstein SD. Migraine symptoms: results of a survey of self-reported migraineurs. Headache J Head Face Pain. 1995;35 (7):387-396. doi:10.1111/j.1526-4610.1995.hed3507387.x

50. Burstein R, Noseda R, Borsook D. Migraine: multiple processes, complex pathophysiology. J Neurosci. 2015;35(17):6619-6629. doi:10.1523/JNEUROSCI.0373-15.2015

51. Sullivan MJL, Bishop SR, Pivik J. The pain catastrophizing scale: development and validation. Psychol Assess. 1995;7(4):524-532. doi:10.1037/1040-3590.7.4.524

52. Rosenstiel AK, Keefe FJ. The use of coping strategies in chronic low back pain patients: relationship to patient characteristics and current adjustment. Pain. 1983;17(1):33-44. 
53. Quartana PJ, Campbell CM, Edwards RR. Pain catastrophizing: a critical review. Expert Rev Neurother. 2009;9(5):745-758. doi:10.1586/ern.09.34

54. Desmond JE, Glover GH. Estimating sample size in functional MRI (fMRI) neuroimaging studies: statistical power analyses. $J$ Neurosci Methods. 2002;118(2):115-128.

55. Moher D, Liberati A, Tetzlaff J, Altman DG; The PRISMA Group. Preferred reporting items for systematic reviews and meta-analyses: the PRISMA statement. PLoS Med. 2009;6(7):e1000097. doi:10.1371/journal.pmed.1000097

56. Armijo-Olivo S, Stiles CR, Hagen NA, Biondo PD, Cummings GG. Assessment of study quality for systematic reviews: a comparison of the cochrane collaboration risk of bias tool and the effective public health practice project quality assessment tool: methodological research: quality assessment for systematic reviews. J Eval Clin Pract. 2012;18(1):12-18. doi:10.1111/j.1365-2753.2010.01516.x

57. Di Pietro F, McAuley JH, Parkitny L, et al. Primary somatosensory cortex function in complex regional pain syndrome: a systematic review and meta-analysis. J Pain. 2013;14(10):1001-1018. doi:10.1016/j.jpain.2013.04.001

58. Martin AR, Aleksanderek I, Cohen-Adad J, et al. Translating state-of-the -art spinal cord MRI techniques to clinical use: a systematic review of clinical studies utilizing DTI, MT, MWF, MRS, and fMRI. NeuroImage Clin. 2016;10:192-238. doi:10.1016/j.nicl.2015.11.019

59. Sullivan MJ, Stanish W, Waite H, Sullivan M, Tripp DA. Catastrophizing, pain, and disability in patients with soft-tissue injuries. Pain. 1998;77(3):253-260.

60. Kadimpati S, Zale EL, Hooten MW, Ditre JW, Warner DO Associations between neuroticism and depression in relation to catastrophizing and pain-related anxiety in chronic pain patients. Eldabe S, ed.. PLoS One. 2015;10(4):e0126351. doi:10.1371/journal.pone.0126351

61. Sullivan MJL, Thorn B, Rodgers W, Ward LC. Path model of psychological antecedents to pain experience: experimental and clinical findings. Clin J Pain. 2004;20(3):164-173.

62. Blankstein U, Chen J, Diamant NE, Davis KD. Altered brain structure in irritable bowel syndrome: potential contributions of pre-existing and disease-driven factors. Gastroenterology. 2010;138(5):1783-1789. doi:10.1053/j.gastro.2009.12.043

63. Ceko M, Bushnell MC, Fitzcharles M-A, Schweinhardt P. Fibromyalgia interacts with age to change the brain. NeuroImage Clin. 2013;3:249-260. doi:10.1016/j.nicl.2013.08.015

64. Hubbard CS, Khan SA, Keaser ML, Mathur VA, Goyal M, Seminowicz DA. Altered brain structure and function correlate with disease severity and pain catastrophizing in migraine patients. eNeuro. 2014;1(1):e20.14. doi:10.1523/ENEURO.0006-14.2014

65. Schweinhardt P, Kuchinad A, Pukall CF, Bushnell MC. Increased gray matter density in young women with chronic vulvar pain Pain. 2008;140(3):411-419. doi:10.1016/j.pain.2008.09.014

66. Seminowicz DA, Shpaner M, Keaser ML, et al. Cognitivebehavioral therapy increases prefrontal cortex gray matter in patients with chronic pain. J Pain. 2013;14(12):1573-1584. doi:10.1016/j.jpain.2013.07.020

67. Henderson LA, Akhter R, Youssef AM, et al. The effects of catastrophizing on central motor activity. Eur J Pain Lond Engl. 2016;20(4):639-651. doi:10.1002/ejp.781

68. Gupta A, Rapkin AJ, Gill Z, et al. Disease-related differences in resting-state networks: a comparison between localized provoked vulvodynia, irritable bowel syndrome, and healthy control subjects. Pain. 2015;156(5):809-819. doi:10.1097/01.j.pain.0000461289.65571.54

69. Kucyi A, Moayedi M, Weissman-Fogel I, et al. Enhanced medial prefrontal-default mode network functional connectivity in chronic pain and its association with pain rumination. $J$ Neurosci Off $J$ Soc Neurosci. 2014;34(11):3969-3975. doi:10.1523/JNEUROSCI.50 55-13.2014
70. Lazaridou A, Kim J, Cahalan CM, et al. Effects of Cognitive-behavioral Therapy (CBT) on brain connectivity supporting catastrophizing in fibromyalgia. Clin $J$ Pain. 2016:1. doi:10.1097/AJP.0000000000000422.

71. Burgmer M, Petzke F, Giesecke T, Gaubitz M, Heuft G, Pfleiderer B. Cerebral activation and catastrophizing during pain anticipation in patients with fibromyalgia. Psychosom Med. 2011;73(9):751-759. doi:10.1097/PSY.0b013e318236588a

72. Hiramatsu T, Nakanishi K, Yoshimura S, et al. The dorsolateral prefrontal network is involved in pain perception in knee osteoarthritis patients. Neurosci Lett. 2014;581:109-114. doi:10.1016/j.neulet.2014.08.027

73. Hubbard CS, Hong J, Jiang Z, et al. Increased attentional network functioning related to symptom severity measures in females with irritable bowel syndrome. Neurogastroenterol Motil Off J Eur Gastrointest Motil Soc. 2015;27(9):1282-1294. doi:10.1111/nmo.12622

74. Lin C-S, Niddam DM, Hsu M-L, Hsieh J-C. Pain catastrophizing is associated with dental pain in a stressful context. J Dent Res. 2013;92(2):130-135. doi:10.1177/0022034512467804

75. Lloyd D, Findlay G, Roberts N, Nurmikko T. Differences in low back pain behavior are reflected in the cerebral response to tactile stimulation of the lower back. Spine. 2008;33(12):1372-1377. doi:10.1097/BRS.0b013e3181734a8a

76. Lloyd D, Helbig T, Findlay G, Roberts N, Nurmikko T. Brain areas involved in anticipation of clinically relevant pain in low back pain populations with high levels of pain behavior. J Pain. 2016;17 (5):577-587. doi:10.1016/j.jpain.2016.01.470

77. Loggia ML, Berna C, Kim J, et al. The lateral prefrontal cortex mediates the hyperalgesic effects of negative cognitions in chronic pain patients. J Pain. 2015;16(8):692-699. doi:10.1016/ j.jpain.2015.04.003

78. Mathur VA, Moayedi M, Keaser ML, et al. High frequency migraine is associated with lower acute pain sensitivity and abnormal insula activity related to migraine pain intensity, attack frequency, and pain catastrophizing. Front Hum Neurosci. 2016;10. doi:10.3389/fnhum.2016.00489

79. Seminowicz DA, Davis KD. Cortical responses to pain in healthy individuals depends on pain catastrophizing. Pain. 2006;120 (3):297-306. doi:10.1016/j.pain.2005.11.008

80. Kim H-J, Cho C-H, Kang K-T, Chang B-S, Lee C-K, Yeom JS. The significance of pain catastrophizing in clinical manifestations of patients with lumbar spinal stenosis: mediation analysis with bootstrapping. Spine J Off J North Am Spine Soc. 2015;15 (2):238-246. doi:10.1016/j.spinee.2014.09.002

81. David SP, Ware JJ, Chu IM, et al. Potential reporting bias in fMRI studies of the brain. Kilner J, ed. PLoS One. 2013;8(7):e70104. doi:10.1371/journal.pone.0070104

82. Davis KD. Neuroimaging of pain: what does it tell us? Curr Opin Support Palliat Care. 2011;5(2):116-121. doi:10.1097/ SPC.0b013e3283458f96

83. Edwards RR, Campbell C, Jamison RN, Wiech K. The neurobiological underpinnings of coping with pain. Curr Dir Psy chol Sci. 2009;18(4):237-241. doi:10.1111/j.1467-8721.2009. 01643.x

84. Tracey I. Imaging pain. $B r \quad J$ Anaesth. 2008;101(1):32-39. doi:10.1093/bja/aen102

85. Davis KD, Moayedi M. Central mechanisms of pain revealed through functional and structural MRI. J Neuroimmune Pharmacol. 2013;8 (3):518-534. doi:10.1007/s11481-012-9386-8

86. Bushnell MC, Čeko M, Low LA. Cognitive and emotional control of pain and its disruption in chronic pain. Nat Rev Neurosci. 2013;14(7):502-511. doi:10.1038/nrn3516

87. Apkarian AV, Baliki MN, Geha PY. Towards a theory of chronic pain. Prog Neurobiol. 2009;87(2):81-97. doi:10.1016/j. pneurobio.2008.09.018 
88. Fillingim RB, King CD, Ribeiro-Dasilva MC, Rahim-Williams B, Riley JL. Sex, gender, and pain: a review of recent clinical and experimental findings. J Pain. 2009;10(5):447-485. doi:10.1016/j. jpain.2008.12.001

89. Rodriguez-Raecke R, Niemeier A, Ihle K, Ruether W, May A. Structural brain changes in chronic pain reflect probably neither damage nor atrophy. Langguth B, ed. PLoS One. 2013;8(2):e54475. doi:10.1371/journal.pone. 0054475

90. Rodriguez-Raecke R, Niemeier A, Ihle K, Ruether W, May A. Brain gray matter decrease in chronic pain is the consequence and not the cause of pain. $J$ Neurosci. 2009;29(44):13746-13750. doi:10.1523/JNEUROSCI.3687-09.2009

91. Peerdeman KJ, van Laarhoven AI, Peters ML, Evers AWM. An integrative review of the influence of expectancies on pain. Front Psychol. 2016;7. doi:10.3389/fpsyg.2016.01270

92. Brown CA, El-Deredy W, Jones AKP. When the brain expects pain: common neural responses to pain anticipation are related to clinical pain and distress in fibromyalgia and osteoarthritis. Eur J Neurosci. 2014;39(4):663-672. doi:10.1111/ejn.12420

93. Castelnuovo G, Giusti EM, Manzoni GM, et al. Psychological considerations in the assessment and treatment of pain in neurorehabilitation and psychological factors predictive of therapeutic response: evidence and recommendations from the italian consensus conference on pain in neurorehabilitation. Front Psychol. 2016;7:468. doi:10.3389/fpsyg.2016.00468

94. Cathcart S, Winefield AH, Lushington K, Rolan P. Stress and tension-type headache mechanisms. Cephalalgia. 2010;30 (10):1250-1267. doi:10.1177/0333102410362927

95. Chen JY-W, Blankstein U, Diamant NE, Davis KD. White matter abnormalities in irritable bowel syndrome and relation to individual factors. Brain Res. 2011;1392:121-131. doi:10.1016/j. brainres.2011.03.069

96. Cottam WJ, Condon L, Alshuft H, Reckziegel D, Auer DP. Associations of limbic-affective brain activity and severity of ongoing chronic arthritis pain are explained by trait anxiety. NeuroImage. 2016;12:269-276. doi:10.1016/j.nicl.2016.06.022

97. Edwards RR, Cahalan C, Calahan C, Mensing G, Smith M, Haythornthwaite JA. Pain, catastrophizing, and depression in the rheumatic diseases. Nat Rev Rheumatol. 2011;7(4):216-224. doi:10.1038/nrrheum.2011.2

98. Fayed N, Andres E, Rojas G, et al. Brain dysfunction in fibromyalgia and somatization disorder using proton magnetic resonance spectroscopy: a controlled study: brain dysfunction in somatization. Acta Psychiatr Scand. 2012;126(2):115-125. doi:10.1111/j.1600-0447.2011.01820.x

99. Goldenberg DL. The Interface of pain and mood disturbances in the rheumatic diseases. Semin Arthritis Rheum. 2010;40(1):15-31. doi:10.1016/j.semarthrit.2008.11.005

100. Gorczyca R, Filip R, Walczak E. Psychological aspects of pain. Ann Agric Environ Med. 2013;1:23-27.

101. Goswami R, Anastakis DJ, Katz J, Davis KD. A longitudinal study of pain, personality, and brain plasticity following peripheral nerve injury. Pain. 2016;157(3):729-739. doi:10.1097/j.pain.0000000000000430
102. Jensen MP, Gianas A, Sherlin LH, Howe JD. Pain catastrophizing and EEG- $\alpha$ asymmetry. Clin J Pain. 2015;31(10):852-858. doi:10.1097/AJP.0000000000000182

103. Kawamichi H, Kitada R, Yoshihara K, Takahashi HK, Sadato N. Interpersonal touch suppresses visual processing of aversive stimuli. Front Hum Neurosci. 2015;9:164. doi:10.3389/fnhum.2015.00164

104. Knudsen L, Petersen GL, Nørskov KN, et al. Review of neuroimaging studies related to pain modulation. Scand J Pain. 2011;2 (3):108-120. doi:10.1016/j.sjpain.2011.05.005

105. Leung L. Pain catastrophizing: an updated review. Indian J Psychol Med. 2012;34(3):204. doi:10.4103/0253-7176.106012

106. Lieberman G, Shpaner M, Watts R, et al. White matter involvement in chronic musculoskeletal pain. J Pain. 2014;15(11):1110-1119. doi:10.1016/j.jpain.2014.08.002

107. Lunn TH, Frokjaer VG, Hansen TB, Kristensen PW, Lind T, Kehlet H. Analgesic effect of perioperative escitalopram in high pain catastrophizing patients after total knee arthroplasty: a randomized, double-blind, placebo-controlled trial. Anesthesiology. 2015;122 (4):884-894. doi:10.1097/ALN.0000000000000597

108. Morris LD, Grimmer-Somers KA, Spottiswoode B, Louw QA. Virtual reality exposure therapy as treatment for pain catastrophizing in fibromyalgia patients: proof-of-concept study (Study protocol). $B M C$ Musculoskelet Disord. 2011;12(1):85. doi:10.1186/1471-2474-12-85

109. Morris LD, Louw QA, Grimmer KA, Meintjes E. Targeting pain catastrophization in patients with fibromyalgia using virtual reality exposure therapy: a proof-of-concept study. $J$ Phys Ther Sci. 2015;27(11):3461-3467. doi:10.1589/jpts.27.3461

110. Piché M, Chen J-I, Roy M, Poitras P, Bouin M, Rainville P. Thicker posterior insula is associated with disease duration in women with irritable bowel syndrome (IBS) whereas thicker orbitofrontal cortex predicts reduced pain inhibition in both IBS patients and controls. J Pain. 2013;14(10):1217-1226. doi:10.1016/j.jpain.2013.05.009

111. Schmidt K, Forkmann K, Sinke C, Gratz M, Bitz A, Bingel U. The differential effect of trigeminal vs. peripheral pain stimulation on visual processing and memory encoding is influenced by pain-related fear. NeuroImage. 2016;134:386-395. doi:10.1016/j. neuroimage.2016.03.026

112. Simons LE, Pielech M, Cappucci S, Lebel A. Fear of pain in pediatric headache. Cephalalgia. 2015;35(1):36-44. doi:10.1177/ 0333102414534084

113. Shimada A, Baad-Hansen L, Svensson P. Effect of experimental jaw muscle pain on dynamic bite force during mastication. Arch Oral Biol. 2015;60(2):256-266. doi:10.1016/j.archoralbio.2014.11.001

114. Wieser MJ, Gerdes ABM, Greiner R, Reicherts P, Pauli P. Tonic pain grabs attention, but leaves the processing of facial expressions intact-evidence from event-related brain potentials. Biol Psychol. 2012;90(3):242-248. doi:10.1016/j.biopsycho.2012.0 3.019

115. Youssef AM, Macefield VG, Henderson LA. Pain inhibits pain; human brainstem mechanisms. Neuroimage. 2016;124(Pt A):54-62. doi:10.1016/j.neuroimage.2015.08.060 


\section{Supplementary Materials}

Appendix SI Studies found and left out from the systematic review

\begin{tabular}{|c|c|c|}
\hline & Author & Reason \\
\hline I & Brown ${ }^{92}$ & Used electroencephalography (EEG) to measure brain activation \\
\hline 2 & Castelnuovo ${ }^{93}$ & Review \\
\hline 3 & Cathcart $^{93}$ & Review \\
\hline 4 & Chen ${ }^{95}$ & Used DTI to measure structural connectivity \\
\hline 5 & Cottam $^{96}$ & Did not examine neural changes connected directly to PCS, only controlled for PCS scores \\
\hline 6 & Edwards ${ }^{97}$ & Review \\
\hline 7 & Fayed $^{98}$ & Mainly brain metabolites and not brain activity \\
\hline 8 & Goldenberg 99 & Review \\
\hline 9 & Gorczyca ${ }^{100}$ & Review \\
\hline 10 & Goswami ${ }^{101}$ & The number of participants is below 12 \\
\hline 11 & Jensen $^{102}$ & Used EEG to measure brain activation \\
\hline 12 & Kawamichi $^{103}$ & Not relevant in our research (since we looked for potential mechanisms underlying catastrophizing) \\
\hline 13 & Knudsen ${ }^{104}$ & Review \\
\hline 14 & Leung $^{105}$ & Review \\
\hline 15 & Lieberman $^{106}$ & Used DTI to measure structural connectivity \\
\hline 16 & Lunn ${ }^{107}$ & No $f M R I$ in the study \\
\hline 17 & Morris ${ }^{108}$ & Only published a study protocol \\
\hline 18 & Morris ${ }^{109}$ & Did not examine neural changes connected directly to PCS \\
\hline 19 & Piché 110 & Did not examine neural changes connected directly to PCS, only controlled for PCS scores \\
\hline 20 & Quartana $^{53}$ & Review \\
\hline 21 & Schmidt ${ }^{\prime \prime \prime}$ & Did not examine neural changes connected directly to PCS \\
\hline 22 & Simons 112 & Review \\
\hline 23 & Shimada ${ }^{113}$ & Used EEG to measure brain activation \\
\hline 24 & Vase $^{22}$ & Used EEG to measure brain activation \\
\hline 25 & Wieser 114 & Used EEG to measure brain activation \\
\hline 26 & Youssef $^{115}$ & Did not examine neural changes connected directly to PCS \\
\hline
\end{tabular}

Abbreviations: EEG, electroencephalography; PCS, pain catastrophizing scale; fMRI, functional magnetic resonance imaging; DTI, diffusion tensor imaging. 
Appendix S2 Risk of bias appraisal (based on data from ${ }^{56-58}$ )

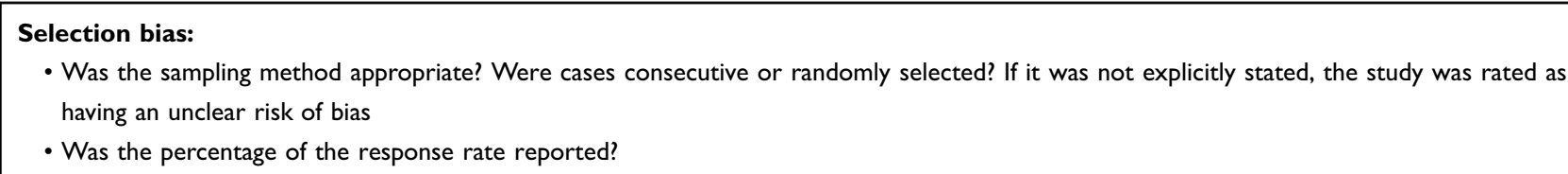

\section{Study design:}

- Was the study design mentioned? What type did they use? Did they choose a method appropriate for the study's aim? (We preferred this question instead of evaluating the study design per se (using the hierarchy of evidence)).

\section{Detection bias:}

- Was the patient group diagnosed according to criteria?

- Did the authors ensure that the controls did not have the patient's condition? (yes if the study uses the same diagnostic tool on the controls too, or describes the control group as " pain-free" or "free of neurological disorders" N/A if there is no control group)

- Did they use matched groups (in race, gender, age, SES, etc.)?

- Were baseline characteristics (age, gender, etc.) clearly described?

\section{Data collection and quality check:}

- Were the acquisition techniques clearly described (scanner type, repetition time, voxel sizes, fov, etc.)?

- Was the task design clearly reported? Were the participants give and instructions? Was the task inside the scanner appropriate? Was the session length appropriate? Were there any the pain ratings?

- Was the task design clearly reported? Were the participants give and instructions? Was the task inside the scanner appropriate? Was the session length appropriate? Were there any the pain ratings?

\section{Drop-out rate described}

- Was the drop-out rate mentioned? (based on the reported numbers, a study was marked as weak if the drop-out rate was more than $40 \%$ )

\section{Confounding variables controlled for}

- Were confounding variables controlled for and reported?

- A study was marked as weak if the authors did not report anything, moderate if the authors controlled for either task [eg, age for gray matter studies] or catastrophizing [eg, depression, neuroticism] relevant variables and strong if the authors controlled for both task and catastrophizing relevant variables

\section{Reporting bias:}

- Did the authors reported the thresholds they used? (was p-value uncorrected or FWE/FDR corrected? Did they use small volume correction at ROls? etc.)

- Were the results clearly reported (with $r$ or $z$ scores)?

- Were all outcomes and groups reported on? (was the result of the study in line with the aims?)

- Studies were marked as weak if they reported uncorrected results or no correction, moderate if they reported FDR/FWE correction but did not report $z$ scores or all outcomes

Abbreviations: SES, socioeconomic status; FWE, family wise error; FDR, false discovery rate; FOV, field of view; ROI, region of interest.

\section{Publish your work in this journal}

The Journal of Pain Research is an international, peer reviewed, open access, online journal that welcomes laboratory and clinical findings in the fields of pain research and the prevention and management of pain. Original research, reviews, symposium reports, hypothesis formation and commentaries are all considered for publication. The manuscript management system is completely online and includes a very quick and fair peer-review system, which is all easy to use. Visit http:// www.dovepress.com/testimonials.php to read real quotes from published authors. 